
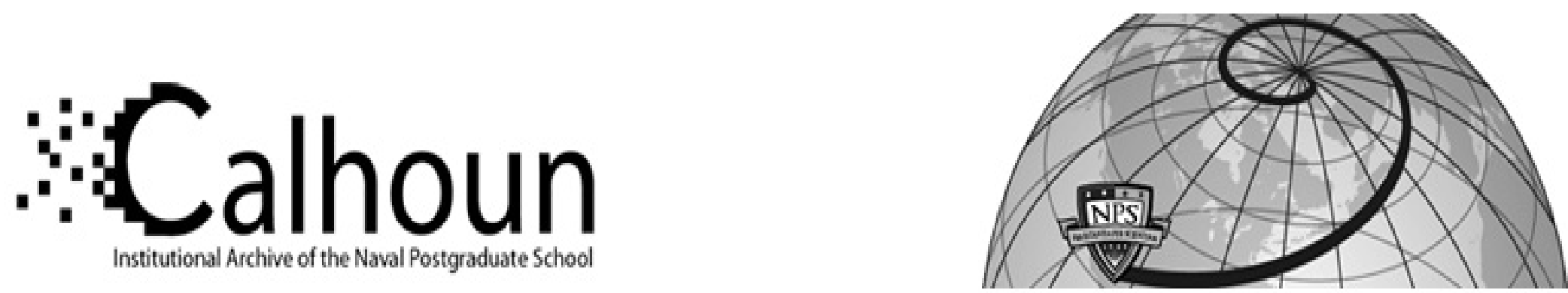

Calhoun: The NPS Institutional Archive DSpace Repository

Comparison of analytic and numerical models with commercially available simulation tools for the prediction of semiconductor freeze-out and exhaustion

Reeves, Derek E.

Monterey, California. Naval Postgraduate School

https://hdl.handle.net/10945/4558

This publication is a work of the U.S. Government as defined in Title 17, United States Code, Section 101. Copyright protection is not available for this work in the United States.

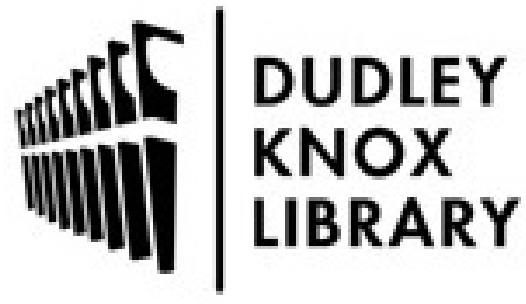

http://www.nps.edu/library
Calhoun is the Naval Postgraduate School's public access digital repository for research materials and institutional publications created by the NPS community. Calhoun is named for Professor of Mathematics Guy K. Calhoun, NPS's first appointed -- and published -- scholarly author.

Dudley Knox Library / Naval Postgraduate School 411 Dyer Road / 1 University Circle Monterey, California USA 93943 


\section{NAVAL POSTGRADUATE SCHOOL Monterey, California}

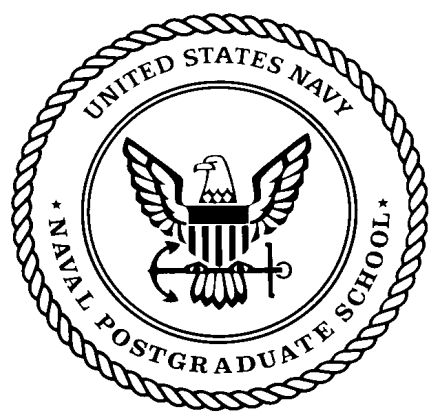

\section{THESIS}

COMPARISON OF ANALYTIC AND NUMERICAL MODELS WITH COMMERCIALLY AVAILABLE SIMULATION TOOLS FOR THE PREDICTION OF SEMICONDUCTOR FREEZEOUT AND EXHAUSTION

by

Derek E. Reeves

September 2002

Thesis Advisor:

Ron J. Pieper

Co-Advisor: Sherif N. Michael

Approved for public release; distribution is unlimited 
THIS PAGE INTENTIONALLY LEFT BLANK 


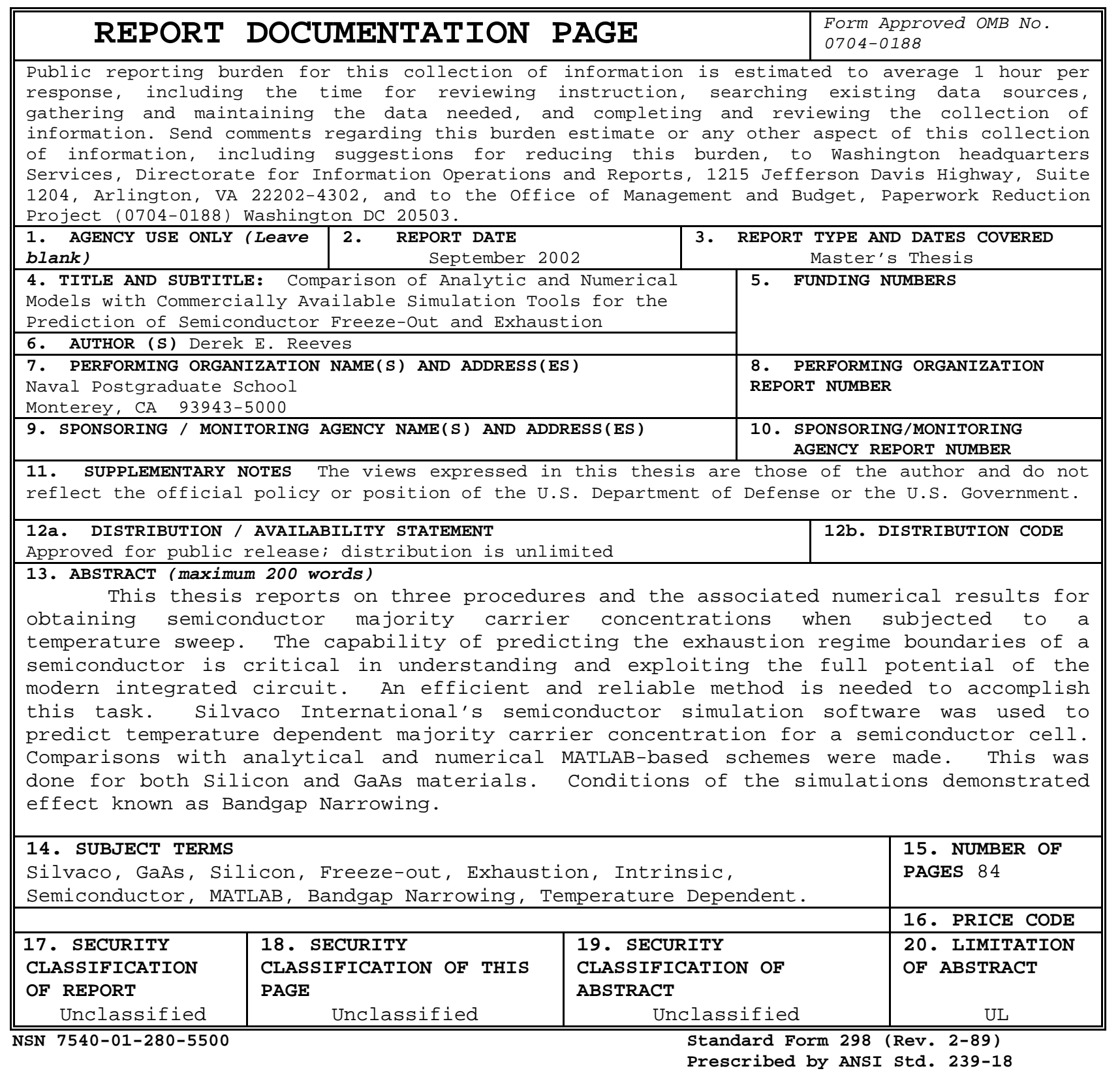


THIS PAGE INTENTIONALLY LEFT BLANK 
Approved for public release; distribution is unlimited

\title{
COMPARISON OF ANALYTIC AND NUMERICAL MODELS WITH COMMERCIALLY AVAILABLE SIMULATION TOOLS FOR THE PREDICTION OF SEMICONDUCTOR FREEZE-OUT AND EXHAUSTION
}

\author{
Derek E. Reeves \\ Lieutenant, United States Navy \\ B.S., Rochester Institute of Technology, 1997
}

Submitted in partial fulfillment of the requirements for the degree of

MASTER OF SCIENCE IN ELECTRICAL ENGINEERING

from the

NAVAL POSTGRADUATE SCHOOL

September 2002

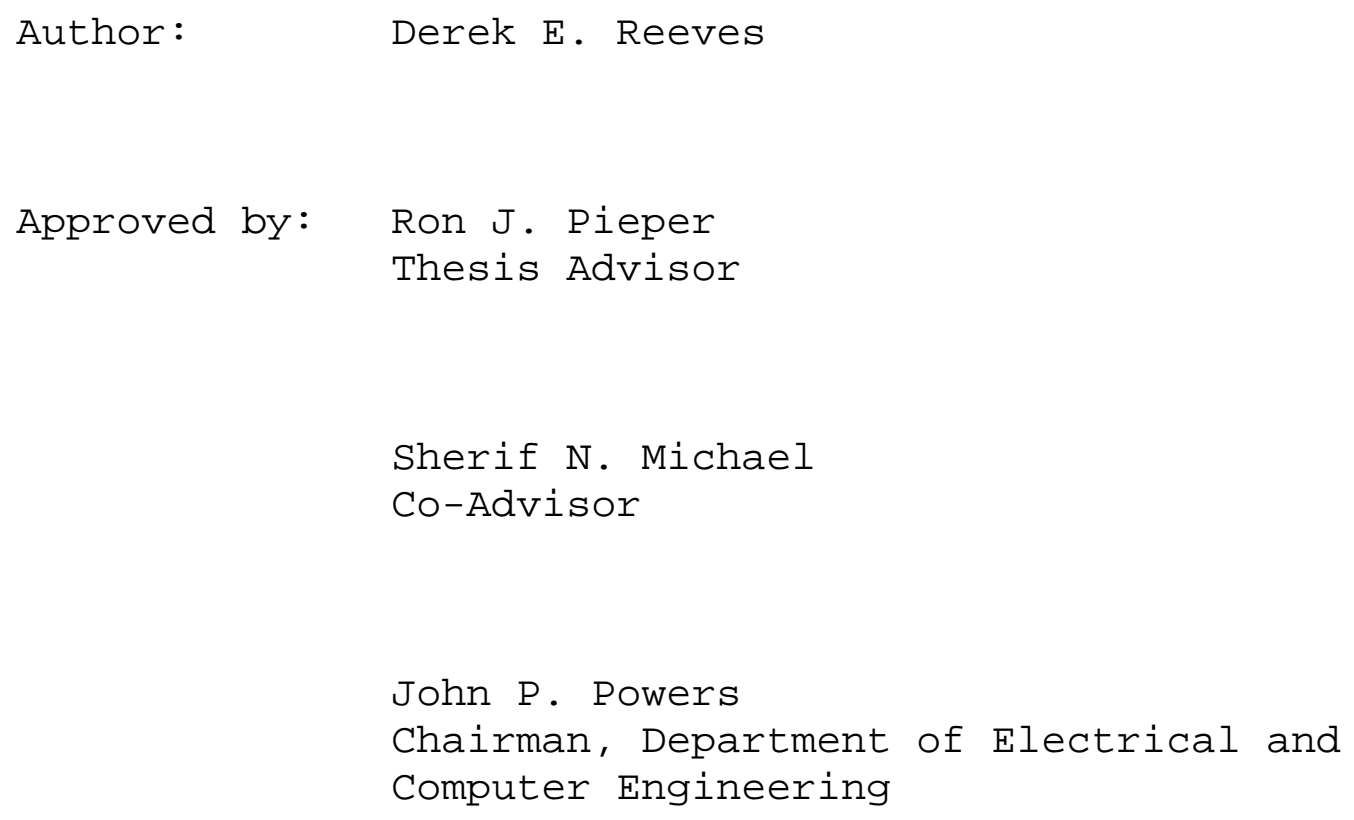


THIS PAGE INTENTIONALLY LEFT BLANK 


\begin{abstract}
This thesis reports on three procedures and the associated numerical results for obtaining semiconductor majority carrier concentrations when subjected to a temperature sweep. The capability of predicting the exhaustion regime boundaries of a semiconductor is critical in understanding and exploiting the full potential of the modern integrated circuit. An efficient and reliable method is needed to accomplish this task. Silvaco International's semiconductor simulation software was used to predict temperature dependent majority carrier concentration for a semiconductor cell. Comparisons with analytical and numerical MATLAB-based schemes were made. This was done for both Silicon and GaAs materials. Conditions of the simulations demonstrated effect known as Bandgap Narrowing .
\end{abstract}


THIS PAGE INTENTIONALLY LEFT BLANK 
TABLE OF CONTENTS

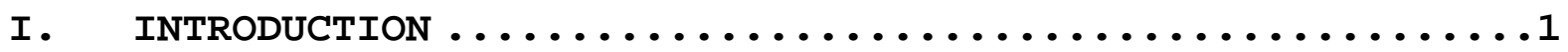

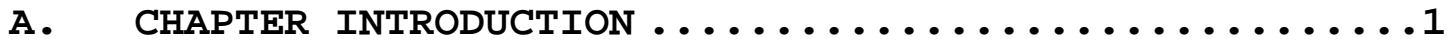

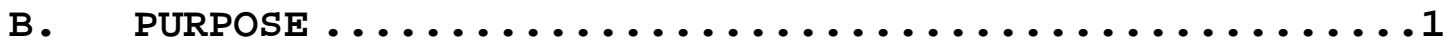

C. INTRODUCTION TO GENERAL TOPIC $\ldots \ldots \ldots \ldots \ldots \ldots \ldots \ldots$

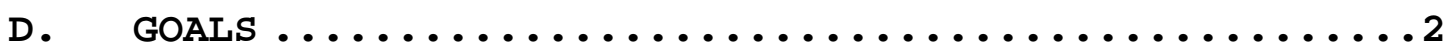

E. THESIS OVERVIEW AND IITERATURE REVIEW .........2

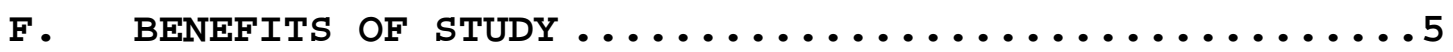

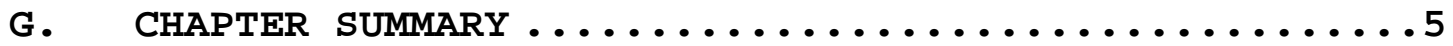

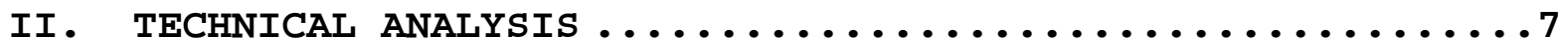

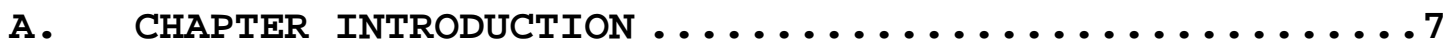

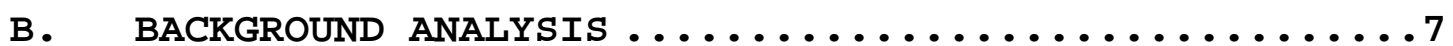

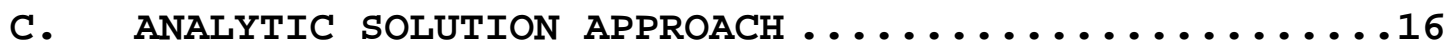

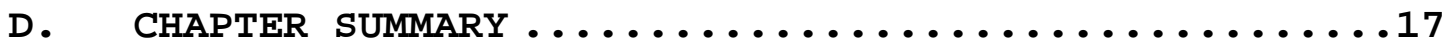

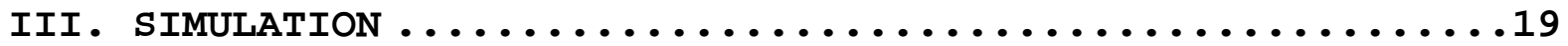

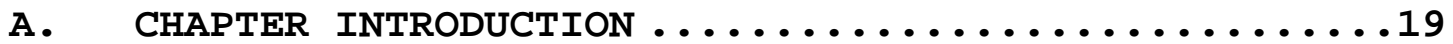

B. SILVACO SIMULATION TOOLS .................. 19

C. DEVELOPMENT OF THE SILVACO SIMULATION PROCESS ...21

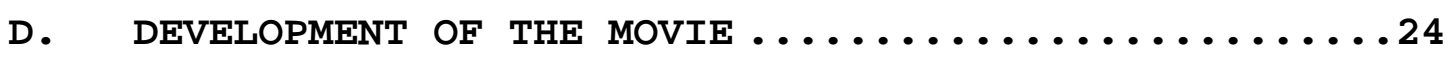

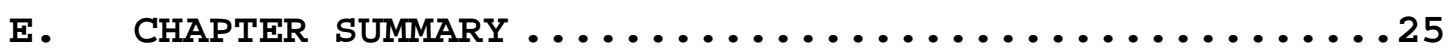

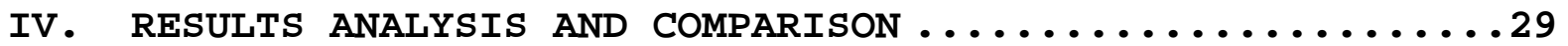

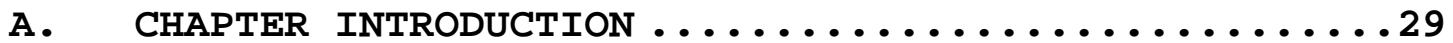

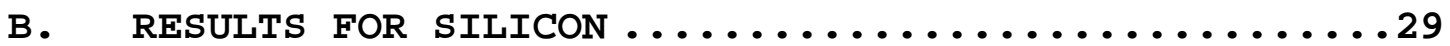

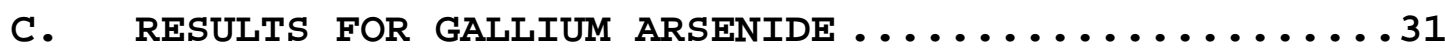

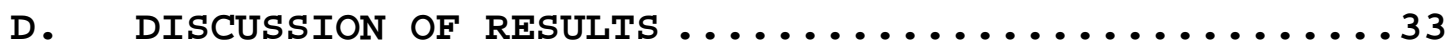

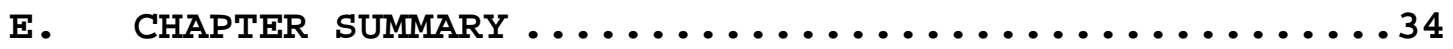

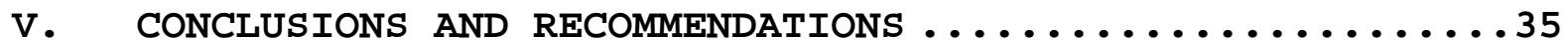

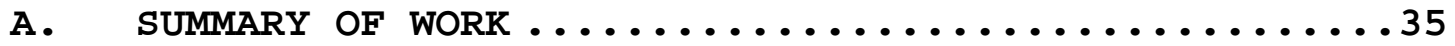

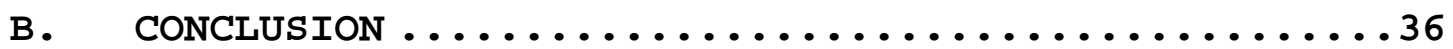

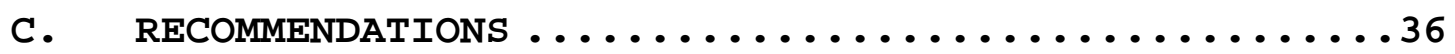

APPENDIX A. INTRODUCTION TO GENERAL SEMICONDUCTOR

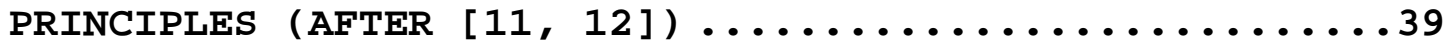

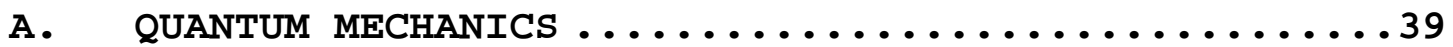

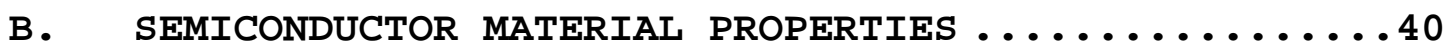

C. CONDUCTION ..................... 42

APPENDIX B. OPERATIONAL GUIDE TO REPRODUCING THE RESULTS

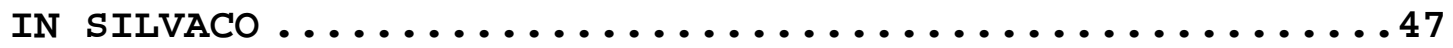

A. OPERATIONAL GUIDE TO REPRODUCING SILVACO DATA

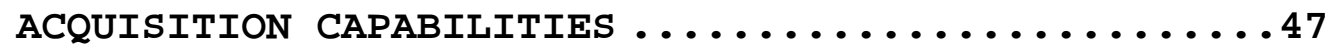

B. DECKBUILD ABBREVIATED SOURCE CODE ...........48 
C. PROCEDURAL GUIDE TO CREATING A MOVIE ...........53 APPENDIX C. PAPER PRESENTED AND ACCEPTED AT THE $45^{\text {TH }}$ IEEE INTERNATIONAL MIDWEST SYMPOSIUM ON CIRCUITS AND

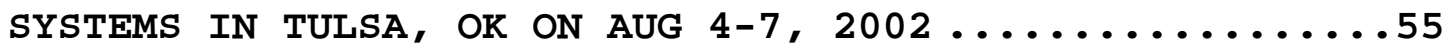

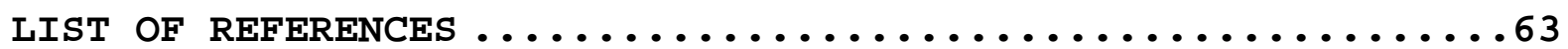

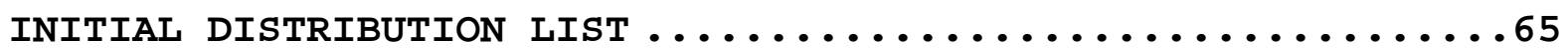




\section{LIST OF FIGURES}

\begin{tabular}{|c|c|c|}
\hline igure & S. 1 & Three Regimes \\
\hline re & $2-1:$ & 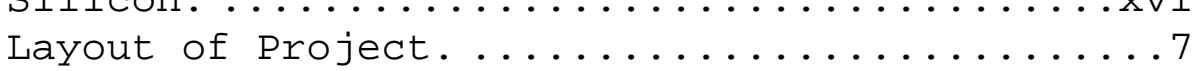 \\
\hline are & $2-2$. & iagram of Bandgap Layout............. \\
\hline re & -1 & ilvaco Interaction Flowchart (From [1])....20 \\
\hline re & $3-2$. & input File Layout (After $[1]) \ldots \ldots \ldots . . . . .21$ \\
\hline e & $3-3$ & lowchart of Silvaco Process.............24 \\
\hline ce & $3-4:$ & Selected Samples of 50 slide Movie $\mathrm{T}=60^{\circ} \mathrm{K} . .26$ \\
\hline re & $3-5$ & Selected Samples of 50 slide Movie $\mathrm{T}=1000^{\circ} \mathrm{K} 27$ \\
\hline re & $4-1$. & omposite Log Plot of three Regimes of Silicon...30 \\
\hline & $4-2$. & site Log Plot of three Regimes of GaAs. .32 \\
\hline 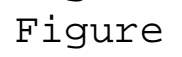 & -1 . & of Photon................40 \\
\hline 1 & -2 . & oped with P (From [13])......43 \\
\hline & & . . . \\
\hline & & \\
\hline
\end{tabular}


THIS PAGE INTENTIONALLY LEFT BLANK 


\section{IIST OF TABLES}

Table ES.1: Silicon Parameters used in Simulations....xvii

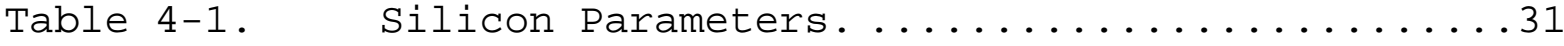

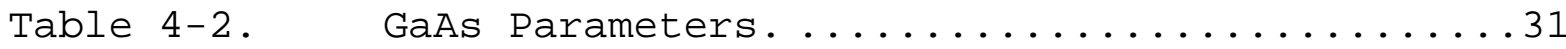


THIS PAGE INTENTIONALLY LEFT BLANK

xii 


\section{ACKNOWLEDGEMENTS}

I would like to give thanks to the following people for helping me complete my thesis. First and foremost, I would like to give thanks to my ancestors for their constant guidance. I would like to thank my parents, Letitia A. Reeves and Eden C. Reeves, for 31 years of love and support that have help me reach this point, and to my siblings, Eden Charles, Denise, and Harriet, for their emotional support and encouragement. I would like to give many thanks to my advisors, Prof. Ronald Pieper and Prof. Sherif Michael, for giving me this great opportunity to work on a meaningful and interesting research topic, and to Prof. Todd Weatherford, for sparking my interest in semiconductors. I would like to thank several key people at Naval Postgraduate School starting with Nathan Beltz, for helping me to learn. Alice Lee, for always providing endearing support, and Nancy Sharrock for aiding me in completing my thesis and being a dear friend. Prof. John Czeiki and Jeff Knight for teaching me the fundamentals of electrical engineering, and last but not least, I would like to thank Heather for helping me through a difficult and challenging time in striving to accomplish this goal. 
THIS PAGE INTENTIONALLY LEFT BLANK

xiv 


\section{EXCEUTIVE SUMMARY}

This thesis reports on three procedures and the associated numerical results for obtaining semiconductor majority carrier concentrations when subjected to a temperature sweep. The capability of predicting the exhaustion regime boundaries of a semiconductor is critical in understanding and exploiting the full potential of the modern integrated circuit. This includes, but not limited to, ICs designed for military hardware as well as specialized extrinsic semiconductors designed for space-based applications. An efficient and reliable method is needed to accomplish the task of predicting exhaustion regime boundaries. Silvaco International's semiconductor simulation software was used to predict temperature dependent majority carrier concentration for a semiconductor cell. Comparisons with analytical and numerical MATLAB-based schemes were made. Conditions of the simulations demonstrated the effect known as Bandgap Narrowing.

The goals of this project are twofold. The first goal is to design a program procedure to temperature sweep a uniform single-doped semiconductor with a two-dimensional Silvaco simulation software[1]. There is a significant challenge to overcome in meeting this goal. Specifically, this is due to a limitation in Silvaco software in that temperature sweeps cannot be performed within a single "run". The second goal was to test the results obtained in Silvaco against one-dimensional analytical and numerical models implemented in MATLAB.

Figure ES contains six plots of $\log _{10}\left(p_{0}\right)$ versus $1000 \times(1 / T)$ for various conditions (with and without band- 
gap narrowing (BGN) and from various sources (numerical, analytic, and Silvacol for the case of doped Silicon. The model for band-gap narrowing [6] used here is:

$$
E_{g}(T)=E_{g o}-\frac{\alpha T^{2}}{(T+\beta)} .
$$

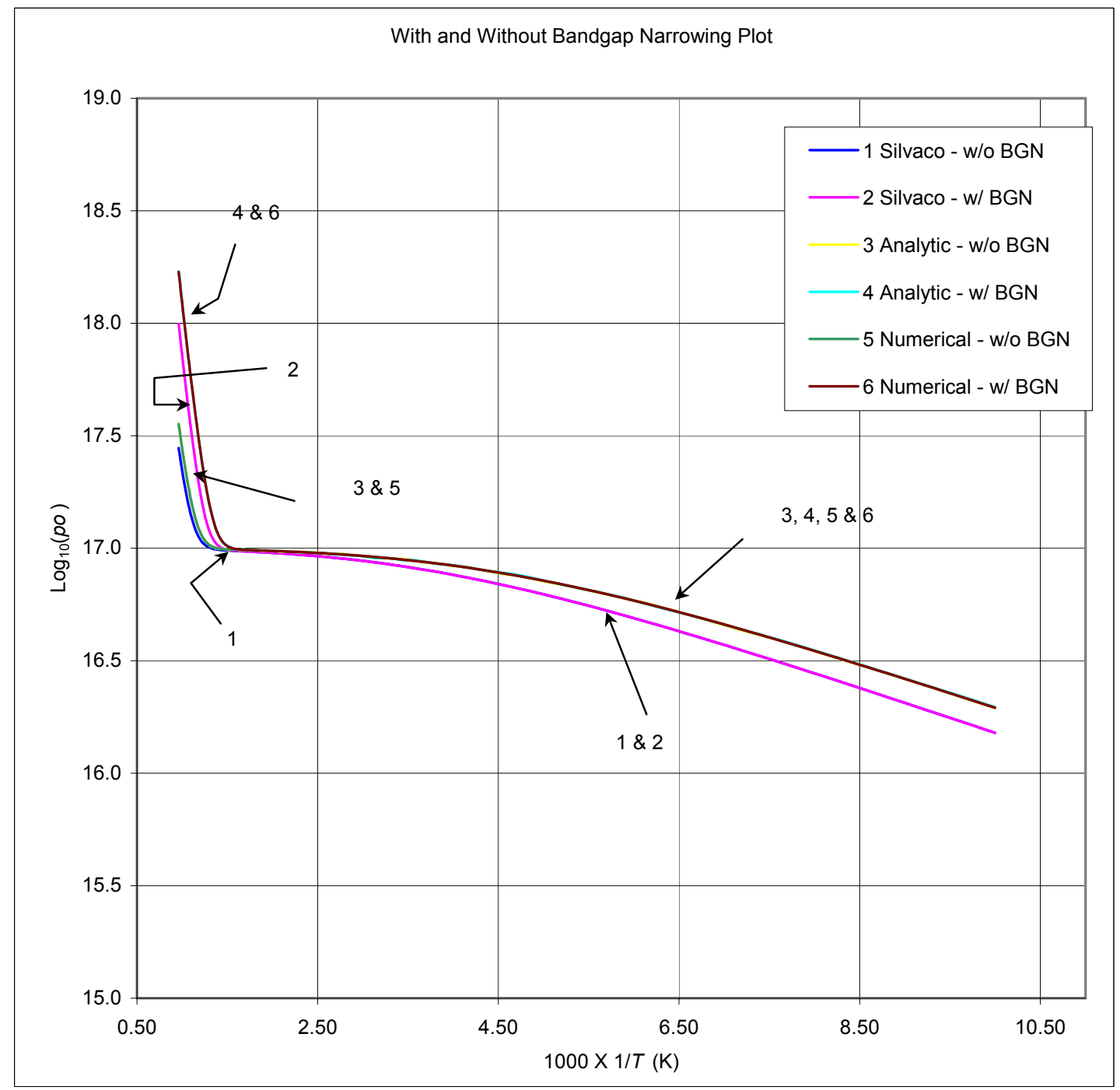

Figure ES.1 Composite Log Plot of Three Regimes of Silicon.

The number codes identify the various curves as per description in the legend of the figure. In Table 1 
recommended values for silicon including BGN parameters $\alpha$ and $\beta$ are provided. Note, as expected, curves from the numerical and analytic methods are indistinguishable, for example, MATLAB-based analytic curve \#4(w/BGN) overlays MATLAB-based numerical curve \#6(w/BGN). Furthermore, as expected the impact of BGN is apparent in the curves at the higher temperatures. The difference between Silvaco predictions and the 1-dimensional modeling efforts are slight but noted for future investigation. Similar analysis was completed for GaAs, with slight variations due to the different material structure and properties. The results for GaAs curves were similar.

\begin{tabular}{|l|l|}
\hline Parameter & Value \\
\hline$E_{a}$ & $0.045 \mathrm{eV}$ \\
\hline$N_{a}$ & $10^{17} \mathrm{\# / \textrm {cm } ^ { 3 }}$ \\
\hline$E_{g \circ}$ & $1.11 \mathrm{eV} \mathrm{(Silicon)}$ \\
\hline$g_{a}$ & 0.00047 \\
\hline$B G N \quad \alpha$ & 636.0 \\
\hline BGN $\beta$ & \\
\hline \multicolumn{2}{|l|}{ Silicon Parameters used in Simulations } \\
\hline
\end{tabular}

TonyPlot was used to create a slideshow movie, to aid in visualization of the process. The structure files from the DeckBuild runs were saved and TonyPlot was used to display the hole/electron concentration of the lattice at temperature steps. This data was then exported into Microsoft office PowerPoint. 


\section{INTRODUCTION}

\section{A. CHAPTER INTRODUCTION}

This first chapter will provide an introduction to the work accomplished in this thesis, a literature review, and a structural overview of the thesis.

\section{B. PURPOSE}

The purpose of this research is to compare the performance of commercial semiconductor two-dimensional simulation tools with one-dimensional analytical and numerical models for freeze-out and exhaustion under a variety of nontrivial conditions.

\section{INTRODUCTION TO GENERAL TOPIC}

Integrated circuits (ICS) are specified to operate between designated temperature limits. The circuit designer selects the doping level or levels and typically assumes that the dopants are approximately 100\% ionized, i.e., exhaustion and temperature are not too high. There can be a significant impact on a plethora of device parameters such as depletions widths and/or FET threshold voltages if the assumption is violated. In the domain where the temperature is too low, the percentage ionization of dopant or dopants will be significantly less than 100\%. The value for the majority carrier concentration is depressed significantly below the design value. On the other hand, if the temperature is too high, the thermal generation effect causes the majority carrier concentration to become excessively higher than the design value in what is called 
the intrinsic temperature regime. The exhaustion regime lies between this two extremes, intrinsic and freeze-out. It is well known that for a multiple impurity dopant process this "simple" three-regime description can be inadequate. What is important to the designer is the plot of the majority carrier versus the temperature or, what is more commonly done, a plot of majority carrier concentration versus the reciprocal of temperature.

\section{GOALS}

The goals of this thesis are twofold. The first goal was to design a program procedure to temperature sweep a uniform single-doped semiconductor using a two-dimensional Silvaco simulation software[1]. There is a significant challenge to overcome in meeting this goal. Specifically, there is a limitation in Silvaco software in that temperature sweeps cannot be performed within a single "run". The second goal was to test the results obtained in Silvaco against one-dimensional analytical and numerical models implemented in MATLAB.

\section{E. THESIS OVERVIEW AND IITERATURE REVIEW}

This research begins in Chapter II with a review of the analysis of the different portions of the project. The analysis in Chapter II serves as the background for the MATLAB numerical and analytic methods. Chapter III is an introduction and description for the Silvaco-based computational tools used to evaluate and compare with the MATLAB-based methods briefly covered in Chapter II. The procedure for modeling of semiconductors in Silvaco and other technical aspects will be discussed. Chapter IV is 
the presentation and discussion of the results obtained from simulations and testing. This data will be used to crosscheck the three approaches to obtaining temperature dependence of majority carrier concentration in singly doped semiconductors. Chapter $V$ is a submittal of conclusions on the study completed and recommendations for further work.

Several topics, which are discussed in the appendices, have been included for completeness. The corresponding information is presented this way, in order to avoid cluttering and overcrowding of the main text. Appendix A provides a brief introduction to general semiconductor principles. Appendix B provides an operational guide to reproducing the results in Silvaco, along with an abbreviated version of the Silvaco DeckBuild source code created for this work. Appendix C includes a pre-print of the paper presented at the $45^{\text {th }}$ IEEE International Midwest Symposium on Circuits and Systems in Tulsa, OK, on Aug 4-7, 2002 .

Currently, commercial software packages, such as available through Silvaco International [1], are well designed to solve the electron/hole transport problem. This type of calculation is usually required to predict the device I-V (current-voltage) characteristics. Surprisingly, using the same package to obtain a temperature dependent plot for majority carrier concentration for a uniform semiconductor requires a somewhat complicated procedure [2]. This thesis presents an efficient and novel way of obtaining this curve from the Silvaco International software and compares the results with a proposed one- 
dimensional single-equation analytic model and a onedimensional numerical model that predicts the temperature dependence for majority concentration in all regimes [3, 4]. To-date, most "analytic" methods for determining dominant features in such plots make use of multiple ad-hoc arguments, which taken one at a time, applies in only two of the three regimes mentioned above $[5,6,7]$. Numerical methods based on one-dimensional analysis provide significant flexibility in terms of making predictions when there are multiple dopants and when taking into account second order effects [4]. It can be argued that numerical methods are generally going to be applicable over a wider range of problems than what can be solved with exact analysis. Two and three-dimensional numerical finite element methods provided in CAD based commercial packages have been widely employed by the engineers in the semiconductor processing industry. The one-dimensional results, both numerical and analytical, are compared with predictions from such a package of tools from Silvaco International [1]. The numerical one-dimensional method is based on solving for zeros in the charge neutrality condition $[5,6,7]$ iteratively for selected temperatures. The numerical algorithm involves a root finding scheme known as the Method of Interval Bisection [8]. The onedimensional MATLAB-based numerical method can be applied on conditions not specifically studied in this thesis such as multiple dopants. Details on the one-dimensional numerical algorithm are not covered in this thesis and interested readers are encouraged to see References [3] and [4]. The analytic expression for the majority carrier temperature 
dependence is based on the well-known solutions to a cubic equation [9].

Band gap narrowing [6] is a well-known effect in which the effective band gap of the semiconductor decreases with increasing temperature. Cases with and without the bandgap narrowing effect were simulated to exhibit the flexibility and consistency of the various approaches taken. Many of the salient features in this thesis have recently been summarized in a short paper [10]. Background information, which appears in Appendix A, was referenced from these sources: [11-15].

\section{F. BENEFITS OF STUDY}

With the proliferation of semiconductor-based applications in modern society, it has become critical to understand the effects of temperature on semiconductors properties. Understanding the nature of temperature sensitivity before production and implementation of a device can reduce cost and risk factors for a final product. For example, the issue is particularly evident in the case of space-based applications, due to the harshness of the environment.

\section{G. CHAPTER SUMMARY}

This Chapter described the general area of investigation, the goals of the thesis, and provided a literature review. In the next chapter, the mathematical formulization, which serves as a basis for one-dimensional analytical and one-dimensional numerical scheme for solving 
for temperature-dependent majority carrier concentration, will be addressed. 


\section{TECHNICAL ANALYSIS}

\section{A. CHAPTER INTRODUCTION}

This chapter provides the background analysis for predictions of temperature dependent majority carrier concentration for both numerical and analytic MATLAB-based approaches (see Figure 2-1).

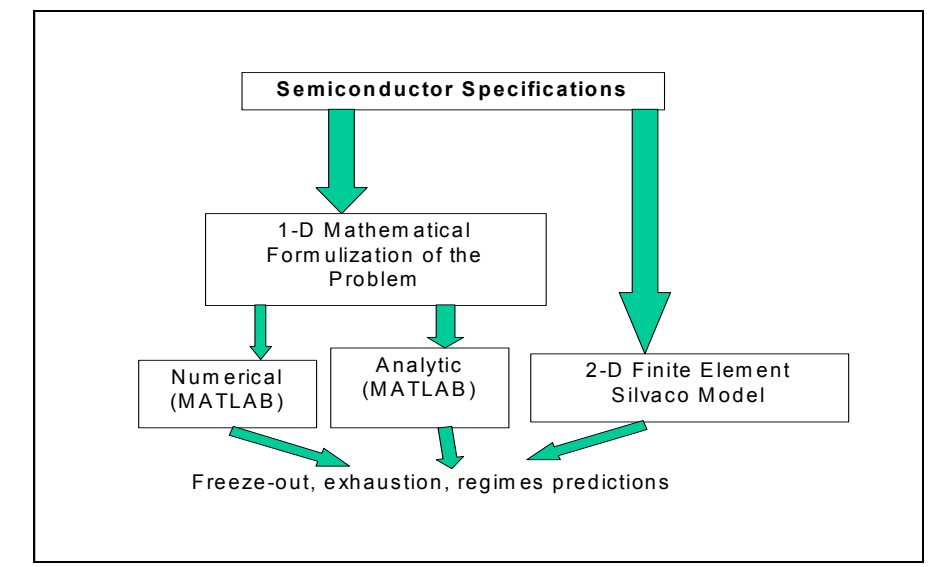

Figure 2-1: $\quad$ Layout of Project.

\section{B. BACKGROUND ANALYSIS}

The following assumptions were made: 1) The semiconductor is spatially uniform, i.e., no spatial variation of the doping and energy bandgap. 2) The semiconductor is in equilibrium. 3) "Degeneracy condition" [6], which is a byproduct of excessive doping, has not occurred. These assumptions were used throughout the simulation for both MATLAB and Silvaco software.

The Fermi-Dirac occupation probability for electrons and holes respectively are: 


$$
F D(E)=\frac{1}{1+e^{\left(E-E_{F}\right) / k T}}
$$

and

$$
\overline{F D(E)}=1-F D(E)=\frac{1}{1+e^{\left(E_{F}-E\right) / k T}}
$$

where $E$ is the energy level defined on electron energy band diagram (see Figure 2-2). The Fermi energy level, $E_{F}$, is where electron and hole occupation probability are both $50 \%, k$ is Boltzman's constant, and $T$ is temperature in Kelvin. Figure 2-2 is a representation of the energy bandgap diagram of a semiconductor. On the right hand side are the levels for the conduction and valance levels, $E_{c}$ and $E_{V}$. The total bandgap size is represented as $E_{g}$, and $\Delta E_{d}$ and $\Delta E_{a}$ are used to represent the difference between the donor $\left(N_{d}\right)$ and acceptor $\left(N_{a}\right)$ levels and the band limits.

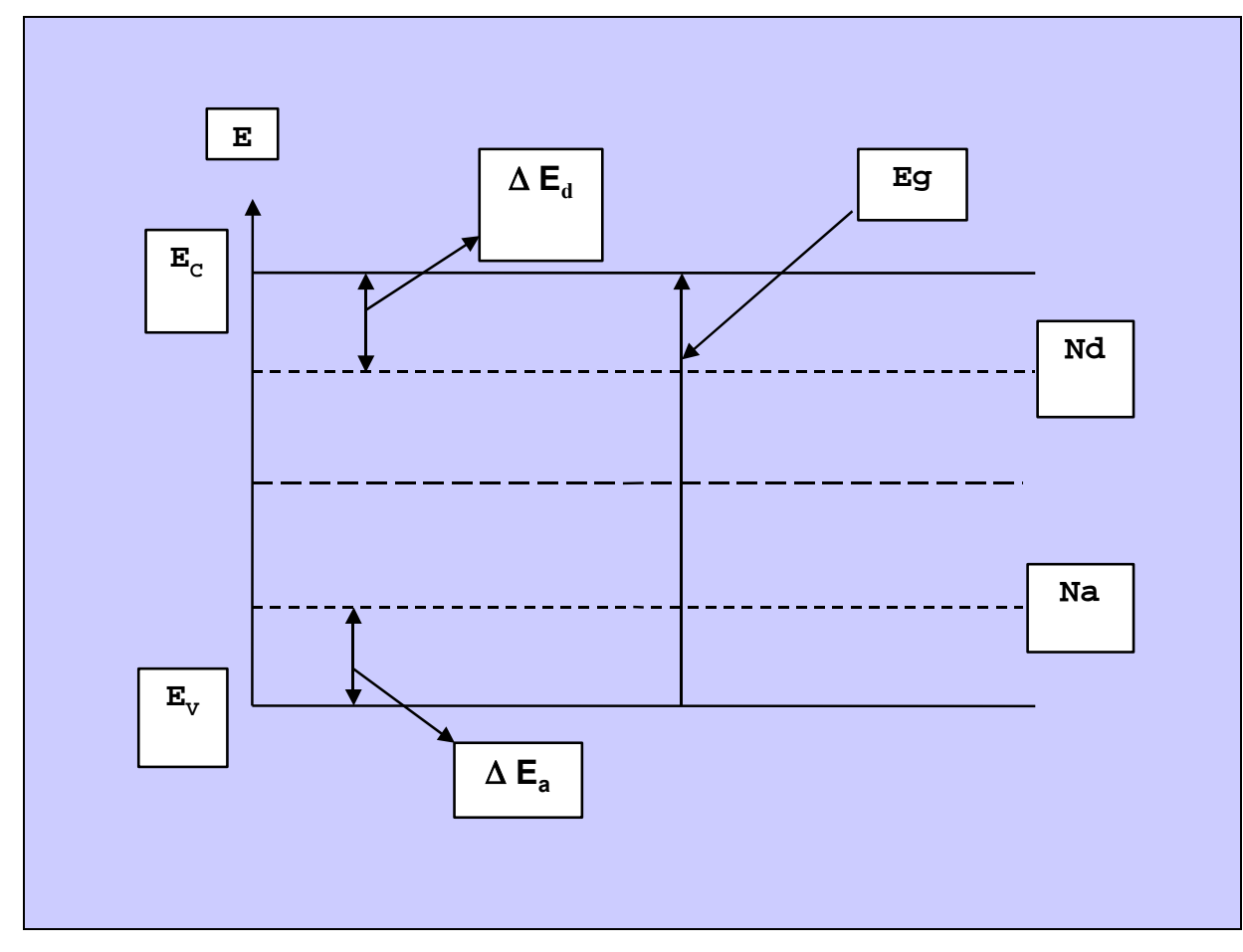

Figure 2-2. Diagram of Bandgap Layout. 
The three-dimensional electron and hole density-ofstates [6] are given by respectively:

$$
\zeta_{s e}(E)=\frac{\sqrt{2}}{\pi}\left(\frac{m_{e}^{*}}{\hbar^{2}}\right)^{3 / 2}\left(E-E_{C}\right)^{1 / 2} H\left(E-E_{C}\right)
$$

and

$$
\zeta_{s h}(E)=\frac{\sqrt{2}}{\pi}\left(\frac{m_{h}^{*}}{\hbar^{2}}\right)^{3 / 2}\left(E_{V}-E\right)^{1 / 2} H\left(E_{V}-E\right)
$$

where $H(x)$ is the Heavy-side function defined as:

$$
H(x)=\left\{\begin{array}{cc}
1 & x \geq 0 \\
0 & \text { otherwise }
\end{array}\right.
$$

and $E_{C}, E_{V}$ are conduction and valance band edges defined on Figure 2-2, $m_{e}^{*}, m_{h}^{*}$ are the effective masses for electrons and holes at those band edges, and $\hbar$ is short-hand notation Planck's constant divided by $2 \pi$.

The density of electron concentration in electron energy space is then obtained from combining Eqs. (2.1) and $(2.2):$

$$
\zeta_{e}(E)=\frac{d n_{0}}{d E}=\zeta_{s e}(E) \star F D(E)
$$

and, similarly, for holes using (2.2) and (2.3):

$$
\zeta_{h}(E)=\frac{d p_{0}}{d E}=\zeta_{s h}(E) \star \overline{F D(E)}
$$

which is the density of hole-concentration in electron energy space.

The equilibrium concentrations of electrons and holes are compactly expressed as: 


$$
n_{0}\left(E_{F}\right)=\int_{-\infty}^{\infty} \zeta_{e}(E) d E
$$

and

$$
p_{0}\left(E_{F}\right)=\int_{-\infty}^{\infty} \zeta_{h}(E) d E
$$

where implicit dependence on Fermi energy level is apparent through substitutions of Eqs. (2.1) through (2.7), into $\mathrm{Eq} \cdot(2.8)$. The transformations, set as $x=\left(E-E_{c}\right) / k T$ for evaluation of $n_{0}\left(E_{F}\right)$ and set as $x=\left(E_{V}-E\right) / k T$ for evaluation of $p_{0}\left(E_{F}\right)$, leads to:

$$
n_{0}\left(E_{F}\right)=\frac{\sqrt{2}}{\pi^{2}}\left(\frac{m_{e}{ }^{*} k T}{\hbar^{2}}\right)^{3 / 2} \frac{\sqrt{\pi}}{2}\left[\frac{2}{\sqrt{\pi}} \int_{0}^{\infty} \frac{x^{1 / 2} d x}{1+e^{x-\eta}}\right]
$$

and

$$
p_{0}\left(E_{F}\right)=\frac{\sqrt{2}}{\pi^{2}}\left(\frac{m_{h}{ }^{\star} k T}{\hbar^{2}}\right)^{3 / 2} \frac{\sqrt{\pi}}{2}\left[\frac{2}{\sqrt{\pi}} \int_{0}^{\infty} \frac{x^{1 / 2} d x}{1+e^{x-\gamma}}\right]
$$

where

$$
\eta=\left(E_{F}-E_{C}\right) / k T
$$

and

$$
\gamma=\left(E_{V}-E_{F}\right) / k T
$$

The expressions defined by (2.10) and (2.11) can be simplified using a standard approximation [6] as shown in the next paragraph.

The Fermi integral is given by [6]:

$$
F_{1 / 2}(y)=\frac{2}{\sqrt{\pi}} \int_{0}^{\infty} \frac{x^{1 / 2} d x}{1+e^{(x-y)}} .
$$


For $y \leq-3$ :

$$
F_{1 / 2}(y) \approx e^{y}\left(\frac{2}{\sqrt{\pi}} \int_{0}^{\infty} e^{-x} x^{1 / 2} d x\right)=e^{y} .
$$

This approximation applied in the context of evaluation of carrier concentrations, is known as the Boltzman's Approximation. In physical terms, the applicability of the approximation depends on the Fermi level being located in the bandgap and not within $3 k T$ of the conduction and valance band edges.

$$
\begin{aligned}
& \text { After combining (2.10) thru (2.15) it follows that: } \\
& \qquad n_{0}\left(E_{F}\right)=N_{C} e^{\left(E_{F}-E_{C}\right) / k T} \chi_{n}
\end{aligned}
$$

and

$$
p_{0}\left(E_{F}\right)=N_{V} e^{\left(E_{V}-E_{F}\right) / k T} \chi_{p}
$$

where effective electron and hole density of states are respectively:

$$
N_{C}=2\left(\frac{2 \pi m_{e}{ }^{*} k T}{\hbar^{2}}\right)^{3 / 2}
$$

and

$$
N_{V}=2\left(\frac{2 \pi m_{h}{ }^{*} k T}{\hbar^{2}}\right)^{3 / 2}
$$

and $\chi_{n}$ and $\chi_{p}$ are degeneracy factors described in the next paragraph.

The electron and hole concentration degeneracy factors [3] are:

$$
\chi_{n} \equiv F_{1 / 2}(\eta) / e^{\eta}
$$

for which $\chi_{n} \approx 1$ for $\eta<-3$ and:

$$
\chi_{p} \equiv F_{1 / 2}(\gamma) / e^{\gamma}
$$


for which $\chi_{p} \approx 1$ for $\gamma<-3$.

If the Boltzman's Approximation can be applied, the usual case if the doping is not too heavy, then $\chi_{n} \sim 1$ and/or $\chi_{p} \sim 1$. The standard Boltzman's approximation form for the equilibrium carrier concentrations is recovered. Specifically

$$
n_{0} \approx N_{C} e^{\left(E_{F}-E_{C}\right) / k T}
$$

and

$$
p_{0} \approx N_{V} e^{\left(E_{V}-E_{F}\right) / k T}
$$

Equations (2.22) and (2.23) can be taken as exact under intrinsic conditions, i.e., no impurity dopant employed.

Two rules are needed to define the intrinsic condition. These rules predict the Fermi level under intrinsic conditions, $E_{F i}$, and the extrinsic carrier concentration, $n_{i}$. Under intrinsic conditions, electron and holes are created in pairs and therefore $n_{i}=n_{o}=p_{o}$. The application using (2.22) and (2.23) leads to:

$$
n_{i}=\sqrt{n_{0} p_{0}}=\sqrt{N_{C} N_{V}} e^{-\left(E_{C}-E_{V}\right) / 2 k T}
$$

and after setting $E_{F}$ to $E_{F i}$ and solving, one determines:

$$
E_{F i}=k T \ln \left(\frac{N_{V}}{N_{C}}\right)+\frac{\left(E_{C}+E_{V}\right)}{2} .
$$

Equations (2.24) and (2.25) can be expressed in term of the bandgap, $E_{g}$, which is represented on Figure 2-2 as the energy difference between the conduction and valence band. If the valance band is used as a reference energy level, i.e., $E_{V}=0$ as represented on Figure 2-2, $E_{g}$ is then: 


$$
E_{g}=\frac{E_{C}-E_{V}}{2}=\frac{E_{C}+E_{V}}{2}
$$

and it follows [6] that Eqs. (2.22) and (2.23) can be expressed in terms of $n_{i}$ and $E_{F i}$ as:

$$
p_{0} \simeq n_{i} e^{\left(E_{F I}-E_{F}\right) / k T}
$$

and

$$
n_{0} \simeq n_{i} e^{\left(E_{F}-E_{F i}\right) / k T}
$$

Equations. (2.27) and (2.28) are the equilibrium carrier concen-trations.

Substitutional impurities can exist in one of two states. Specifically, either the neutral state or an ionized state can exist. The basic rules that govern donor and acceptor ionizations [6] are:

$$
\frac{N_{d}{ }^{0}}{N_{d}{ }^{+}}=g_{d} e^{\left(\frac{E_{F}-E_{d}}{k T}\right)}
$$

and

$$
\frac{N_{a}^{-}}{N_{a}{ }^{0}}=\frac{1}{g_{a}} e^{\left(\frac{E_{F}-E_{a}}{k T}\right)},
$$

where subscript ' $d$ ' is for donor and ' $a$ ' is for acceptor, $N_{d}{ }^{0}$ and $N_{a}{ }^{0}$ refer to neutral donor and acceptor impurity concentrations respectively, $N_{d}^{+}$and $N_{a}^{-}$refer to ionized donor and acceptor concentrations, and $g_{d}$ and $g_{a}$ are occupation indices. There are electron spin-based arguments $[6,12]$ that indicate in most semiconductors $g_{a}=4$ and $g_{d}=2$. The fraction of ionization can be established via several observations. First, the sum of ionized parts, plus neutral must be added to the total impurity used, specifically for donors: 


$$
N_{d}=N_{d}^{0}+N_{d}^{+}
$$

and for acceptors:

$$
N_{a}=N_{a}^{0}+N_{a}^{-}
$$

After dividing both sides of Eq. (2.31) by $N_{d}$, it is found that:

$$
\left(\frac{N_{d}^{+}}{N_{d}}\right)^{-1}=1+\frac{N_{d}^{0}}{N_{d}^{+}}
$$

where $\mathrm{N}_{\mathrm{d}}$ and $\mathrm{N}_{\mathrm{a}}$ are identified on Figure 2-2.

Similarly, after dividing both sides of Eq. (2.32) by $N_{a}$, it is found that:

$$
\left(\frac{N_{a}^{-}}{N_{a}}\right)^{-1}=1+\frac{N_{a}^{0}}{N_{a}^{-}} .
$$

Therefore, substitution of Eqs. (2.31 and 2.32) into Eqs. (2.33 and 2.34) leads to:

$$
\frac{N_{d}^{+}}{N_{d}}=\frac{1}{1+g_{d} e^{\left(\frac{E_{F}-E_{d}}{k T}\right)}}
$$

and

$$
\frac{N_{a}^{-}}{N_{a}}=\frac{1}{1+g_{a} e^{\left(\frac{E_{a}-E_{F}}{k T}\right)}}
$$

which provides the guidelines for Fermi energy level dependent degrees of ionization.

Donor energy levels are typically cited using the conduction band as the reference. For similar reasons, the acceptor energy levels are typically cited using the valence band as a reference [3]:

$$
E_{d}=E_{C}-\Delta E_{d}
$$


and

$$
E_{a}=\Delta E_{a}
$$

which is consistent with Figure 2-2.

Assuming we have $M_{d}$, mono-valent donors, and $M_{a}$, monovalent acceptors, the conditions of local charge neutrality exist. This is based on assumptions \#1 and \#2. For the condition:

$$
P\left(T, E_{F}\right)=p_{0}-n_{0}+\sum_{1=1}^{M_{d}}\left(N_{d}^{+}\right)_{1}-\sum_{l=1}^{M_{a}}\left(N_{a}^{-}\right)_{1}=0
$$

where there is an implicit dependence on both $E_{F}$ and the temperature within each term of Eq. (2-22). The dependencies are obtained from Eqs.(2.35 to 2.38). For a specified temperature, Eq. (2.39) results in a non-linear expression in $Z$, where $Z=e^{\left(E_{F i}-E_{F}\right) / k T}$. At a specified $Z$, the carrier concentrations can be predicted from:

$$
n_{0}=\frac{n_{i}}{Z}
$$

and

$$
p_{0}=n_{i} Z
$$

which is an equivalent revised version of Eqs. (2.27) and $(2.28)$.

Making use of the Fermi probability distributions to predict ionization levels of the donors and acceptors, specifically this requires the substitution of Eqs. (2.35, 2.36, 2.40, and 2.41) into Eq. (2.39) and dividing by the intrinsic concentration which leads to a condition on $P(Z, T):$

$$
P(Z)=0=Z+\sum_{1=1}^{M_{d}} \frac{\bar{N}_{d_{1}}}{1+K_{d_{1}} Z^{-1}}-Z^{-1}-\sum_{l=1}^{M_{a}} \frac{\bar{N}_{a_{1}}}{1+K_{a_{1}} Z} .
$$


The over-bar notation indicates division by $n_{i}$. The temperature dependent ionization factors:

$$
K_{d_{1}} \equiv g_{d} e^{\left(E_{F i}-E_{d_{1}}\right) / k T}
$$

and

$$
K_{a_{1}} \equiv g_{a} e^{\left(E_{a_{1}}-E_{F i}\right) / k T}
$$

serves as a gauge for degrees of ionization of the associated impurity. In summary, Eqs. (2.42 to 2.44) serve as a mathematical basis for both the analytic and numerical $[3,4]$ MATLAB-based approaches.

\section{ANALYTIC SOLUTION APPROACH}

This situation is described by the special case that $M_{a}=1$ and $M_{d}=0$. The summation subscript in Eq. (2.42) can be dropped to lighten the notation. Straightforward algebra leads to a cubic equation in $z$ :

$$
z^{3}+\frac{1}{K_{a}} z^{2}-\left(\frac{\bar{N}_{a}}{1+K_{a}}\right) z-\frac{1}{K_{a}}=0 .
$$

The corresponding result for a single impurity N-type is exactly the same form, producing a cubic in $Y=1 / Z$ with revised coefficients obtained by letting the ' $a$ ' subscript (for acceptor) be replaced with the ' $d$ ' subscript (for donor). The solution can be defined in terms of coefficients for the reference cubic equation:

$$
x^{3}+a_{1} x^{2}+a_{2} x+a_{3}=0 \text {. }
$$

To facilitate representation of a solution, the following intermediate parameterization of the problem is commonly taken [9]:

$$
Q=\left(3 a_{2}-a_{1}^{2}\right) / 9
$$


and

$$
R=\left(9 a_{1} a_{2}-27 a_{3}-2 a_{1}^{3}\right) / 54
$$

and

$$
D \equiv Q^{3}+R^{2}
$$

where, $D$, which is referred to as the "discriminant" for the cubic problem, will dictate the type of solutions possible. As per the fundamental theorem of algebra there will be three roots. For $D$ negative, all roots are real while for $D$ positive only one root is real while the other two are complex conjugates. If $D$ is zero there will be repeated root. For the problem being evaluated it can be shown using symbolic mathematical methods that $Q$ and $D$ will always be negative. Furthermore, it can then be shown that of the three roots, only one is positive and, therefore, physically acceptable. That root will be predicted by the following formula [9]:

$$
z=x=2 \sqrt{-Q} \cos (\theta / 3)-\frac{1}{3} a_{1}
$$

where $\theta=\cos ^{-1}\left(R / \sqrt{-Q^{3}}\right)$ once $z$ is determined, the majority carrier concentration, $p_{0}$, can be predicted from Eq. (2.41).

\section{CHAPTER SUMMARY}

This Chapter has established the mathematical basis for both MATLAB-based analytical and numerical predictor algorithms for temperature dependence of the majority carrier (see Figure 2-1). In this Chapter the main steps leading to a single equation analytic solution are described. In the next Chapter, the third approach, based 
on the commercially available Silvaco International software, is briefly explained. 


\section{SIMULATION}

\section{A. CHAPTER INTRODUCTION}

For this project, a suite of commercially available simulation tools [1] intended for semiconductor testing was used. The primary objective of the thesis was to generate Silvaco predictions for the temperature dependent majority carrier. A comparison will be made with the MATLAB-based analytical and numerical methods discussed in the previous chapter (see Figure 2-1). The data from all three approaches were then compared graphically using Microsoft Excel. The following sections will discuss the processes developed in the simulation to generate the data for this comparison testing.

\section{B. SILVACO SIMULATION TOOLS}

The simulation in Silvaco was designed using the following modules from the suite: ATLAS, DeckBuild, TonyPlot and DevEdit. The following outline (Figure 3-1) illustrates the interconnecting relationships between the individual modules. 


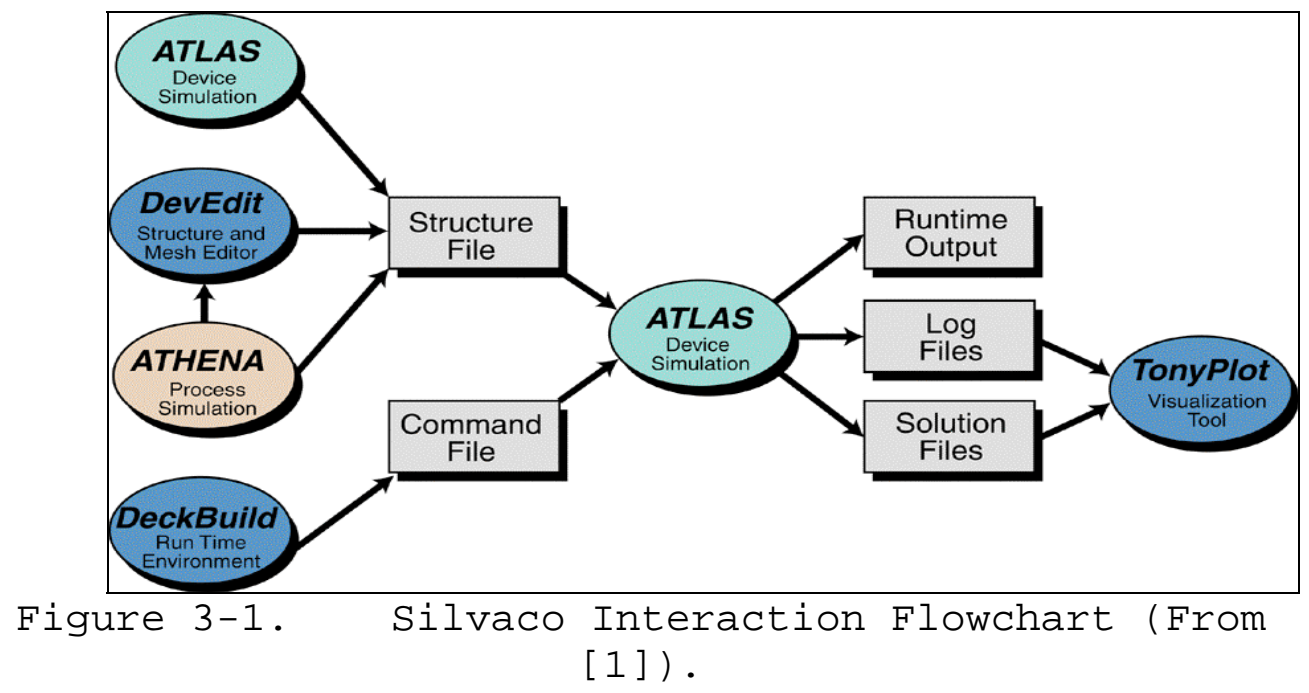

ATLAS is a 2D/3D simulator for semiconductor devices. ATLAS can provide data and insights into the internal physical mechanisms of a device based on predicted electrical behavior. It can either be used as a standalone tool or as a core unit for the Silvaco Virtual Wafer Fabrication environment. This module was used in connection with the other modules to deliver powerful and accurate data on the behavior of semiconductors [1].

DeckBuild is an interactive graphical user interface to provide a user-friendly runtime environment for integration of the different aspects of the Silvaco Software suite. A control window is provided for file creation and control. Many of the features are automated to allow for accurate simulation in a simple to use environment. Tonyplot is the stand-alone program, which can also be referenced in DeckBuild, to display the results [1] .

DevEdit is a device editor which can be used to generate a mesh for the structure designed in DeckBuild and 
ATLAS. A limitation of device simulators prior to DevEdit was inadequate or poor structure meshes. DevEdit's usage was integrated into DeckBuild to allow for a more complete and accurate solution [1].

\section{DEVELOPMENT OF THE SILVACO SIMULATION PROCESS}

In order to develop the overall simulation, the first step was to understand the full scope of the problem and to determine the best features of Silvaco to find a solution. There were many automated features and defaults settings needed to be addressed to ensure that both sets of data were based on the same fundamentals. The DeckBuild coding in Silvaco followed the standard format of the program as illustrated in Figure 3-2:

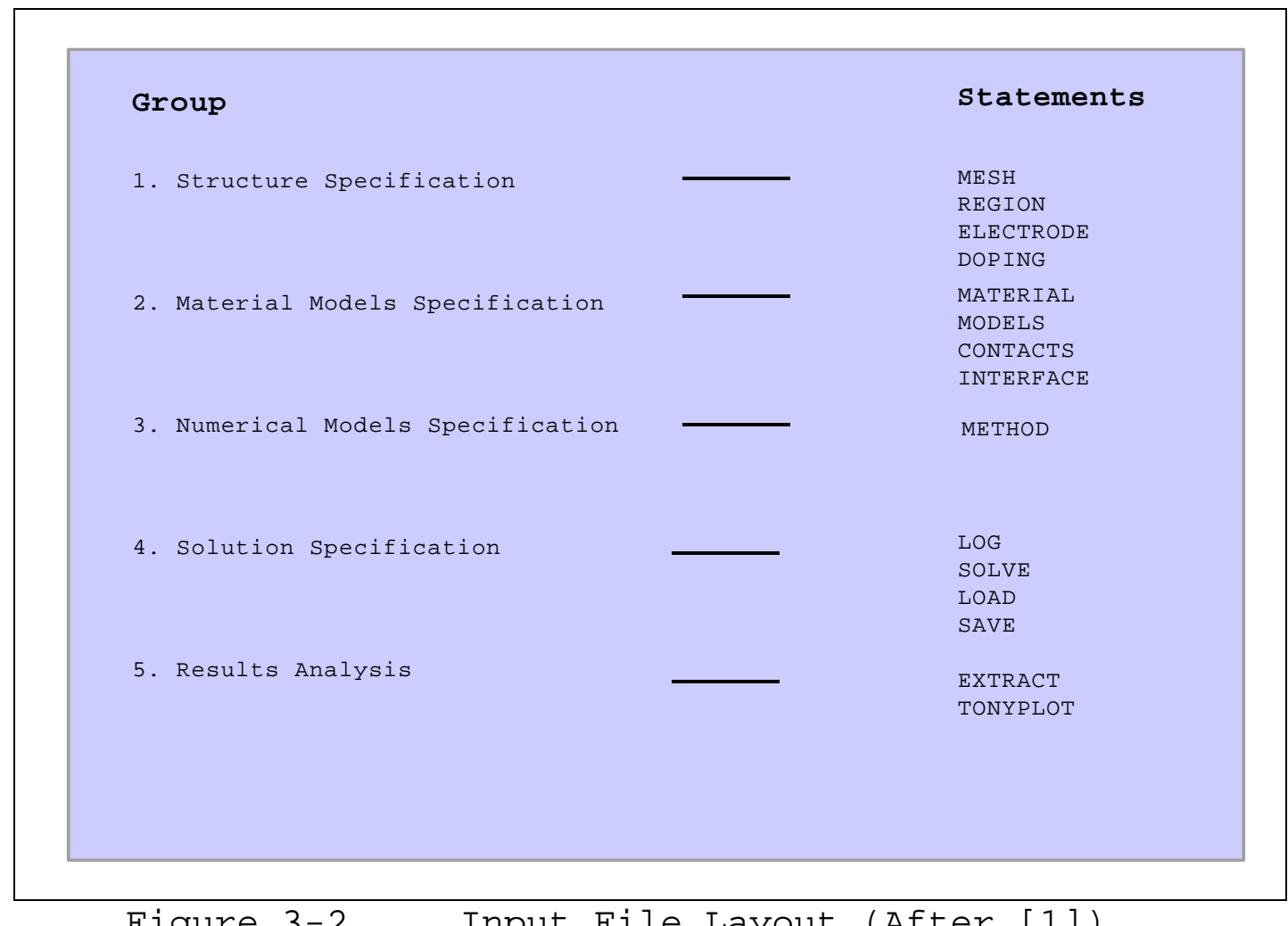

Figure 3-2. Input File Layout (After [1]). 
In Figure 3-2, the left side, listed under Group, outlines how commands are clustered in Silvaco. The right side, listed under statements, catalogs several of the commonly used commands and the order in which they are called.

Several important values were set at the beginning of the DeckBuild code to ensure that default settings were not used. The specified parameters included: the dopant used, the e-alpha $(\alpha)$, e-beta $(\beta)$, and e-band gap $\left(E_{g o}\right)$ values, which were set before initializing the program. As previously stated, the bandgap represents one of the most important parameters of a semiconductor and thus, it was set explicitly in DeckBuild coding. The equation for bandgap narrowing listed in the Silvaco ATLAS Manual [1] is as follows:

$$
E_{g}(T)=E_{g}(300)+\alpha\left[\frac{300^{2}}{300+\beta}-\frac{T^{2}}{T+\beta}\right]
$$

where $T$ is the temperature in degrees Kelvin and the $\alpha$ and $\beta$ parameters are semiconductor material dependent. $E_{g}(300)$ is the energy gap at $300^{\circ} \mathrm{K}$. An equivalent formulization used in the MATLAB-based program is in terms of $E_{g}(0)$ instead of $E_{g}(300)[6,10]$.

The structural dimensions of the device were then set and the contacts were placed with DevEdit. The device material was then set and the doping concentration was also set in DeckBuild. Choosing a model was the next challenging aspect of research since certain DeckBuild coding options would decide the included physical effects. This was needed in order to obtain the most comprehensive solution to compare with the MATLAB-based approaches (see Figure 2-1). 
For the model options in the Silvaco Simulations DeckBuild code, incomplete, conmob, and fermi were chosen, because they covered the widest comprehensive range of temperature-dependent effects. The incomplete portion was used to properly account for freeze-out when there were not sufficient amounts of thermal energy to assume 100\% dopant ionization. The conmob function was used to maintain concentration mobility as per the simple power rule for temperature dependence. The fermi command was used to account for cases of heavily doped materials.

Along with the technical challenges of understanding the correct configuration for modeling a semiconductor, there were the difficulties in manipulating Silvaco due to inherent limitations of the program suite. A major obstacle related to the inability to program a temperature sweep in Silvaco within a single run. A method had to be developed to complete this process in an automated fashion. It was accomplished by replicating single-run code segments within DeckBuild. Each of the code segments was essentially the same, except for the temperature, which was incremented consecutively (see Appendix B). The temperature along with other pertinent data were recorded and logged after each step, which completed the ATLAS run. This process was repeated over a selected temperature range. Stored data could then be exported to a program, such as Microsoft Excel. This process allowed for significant flexibility in selecting the semiconductor parameters. It also allowed for an automation in the process which required very minimal user input. The overall process is illustrated in Figure 3-3. 


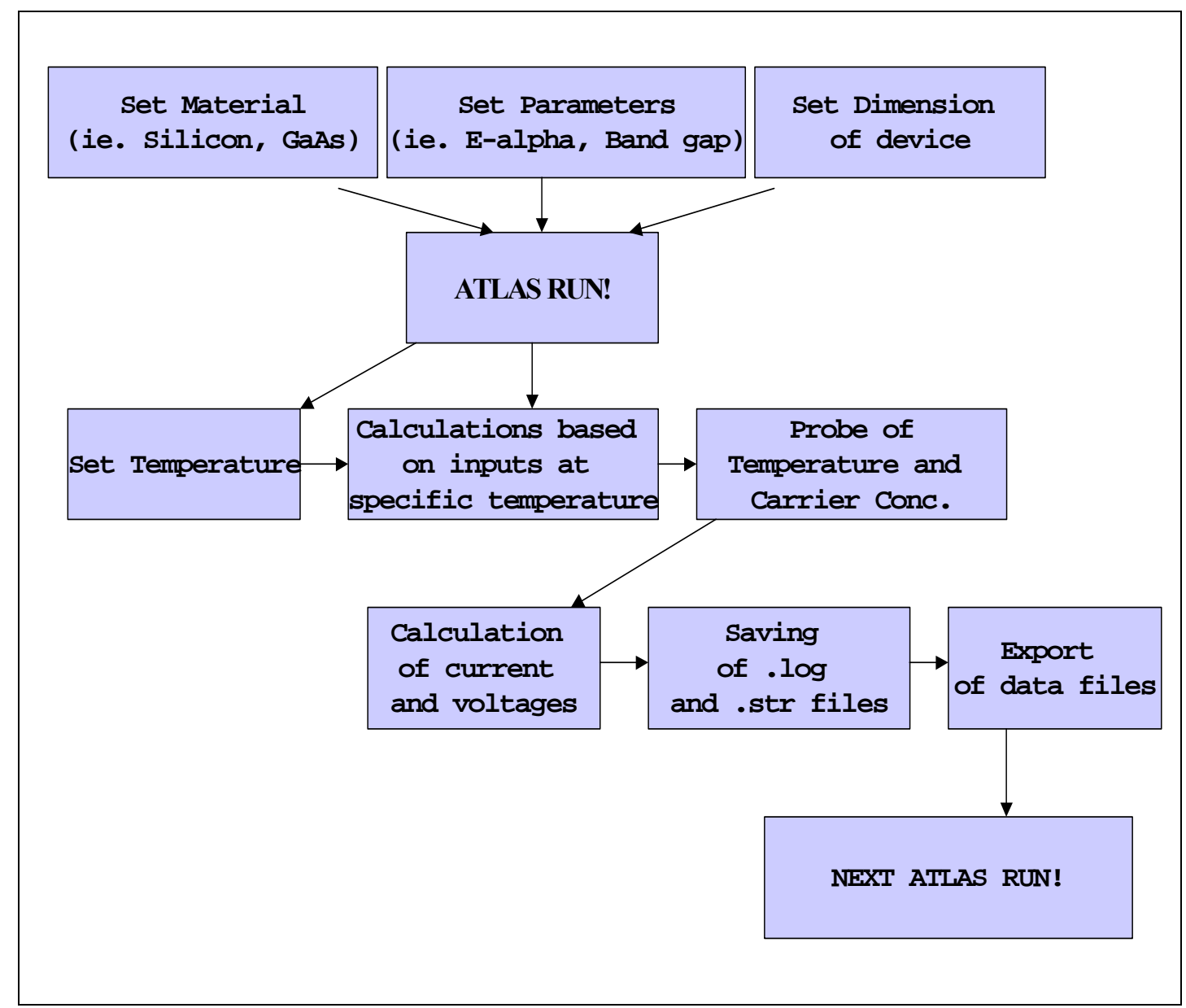

Figure 3-3. Flowchart of Silvaco Process.

\section{DEVELOPMENT OF THE MOVIE}

Tonyplot was used to create a slideshow, to aid in visualization of the process. The structure files from the DeckBuild runs were saved and TonyPlot was used to display the hole/electron concentrations of the lattice over the temperature steps. These data outputs were then exported into other commercial software packages that allowed for graphic file animation, such as Microsoft Office PowerPoint. Two samples of the movie are provided in Figure 3-4 and 3-5. The arrow over the curves on the right side identifies the temperature. The upper half shows a selected 
movie slide when the temperature was approximately $10 \mathrm{~K}$ while the lower half was taken at a much higher temperature of $900 \mathrm{~K}$. Note that colors for the left side are legendencoded for the concentrations of the majority carrier at the corresponding temperature. More details on the procedure for creating a movie are provided in Appendix B.

\section{E. CHAPTER SUMMARY}

This Chapter outlined and explained the technical aspects of utilizing commercially available Silvaco International software for the objectives of this thesis. A more detailed description of this procedure is provided in Appendix B. The next chapter will provide a review and comparison of the Silvaco based results with predictions generated from MATLAB-based analytic and numerical algorithm. 


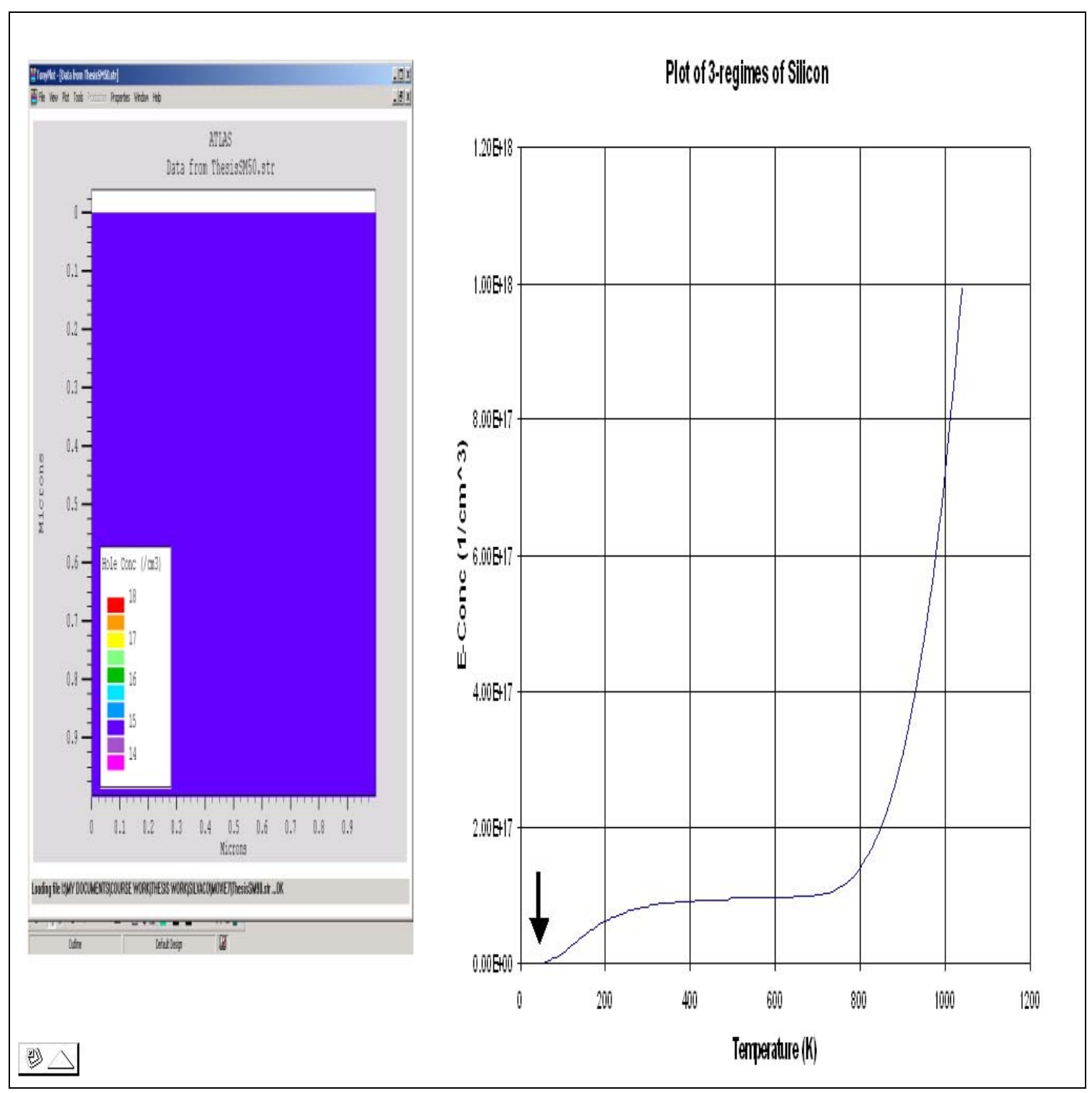

Figure 3-4: Selected Samples of 50 slide Movie $\mathrm{T}=$ $60^{\circ} \mathrm{K}$ 


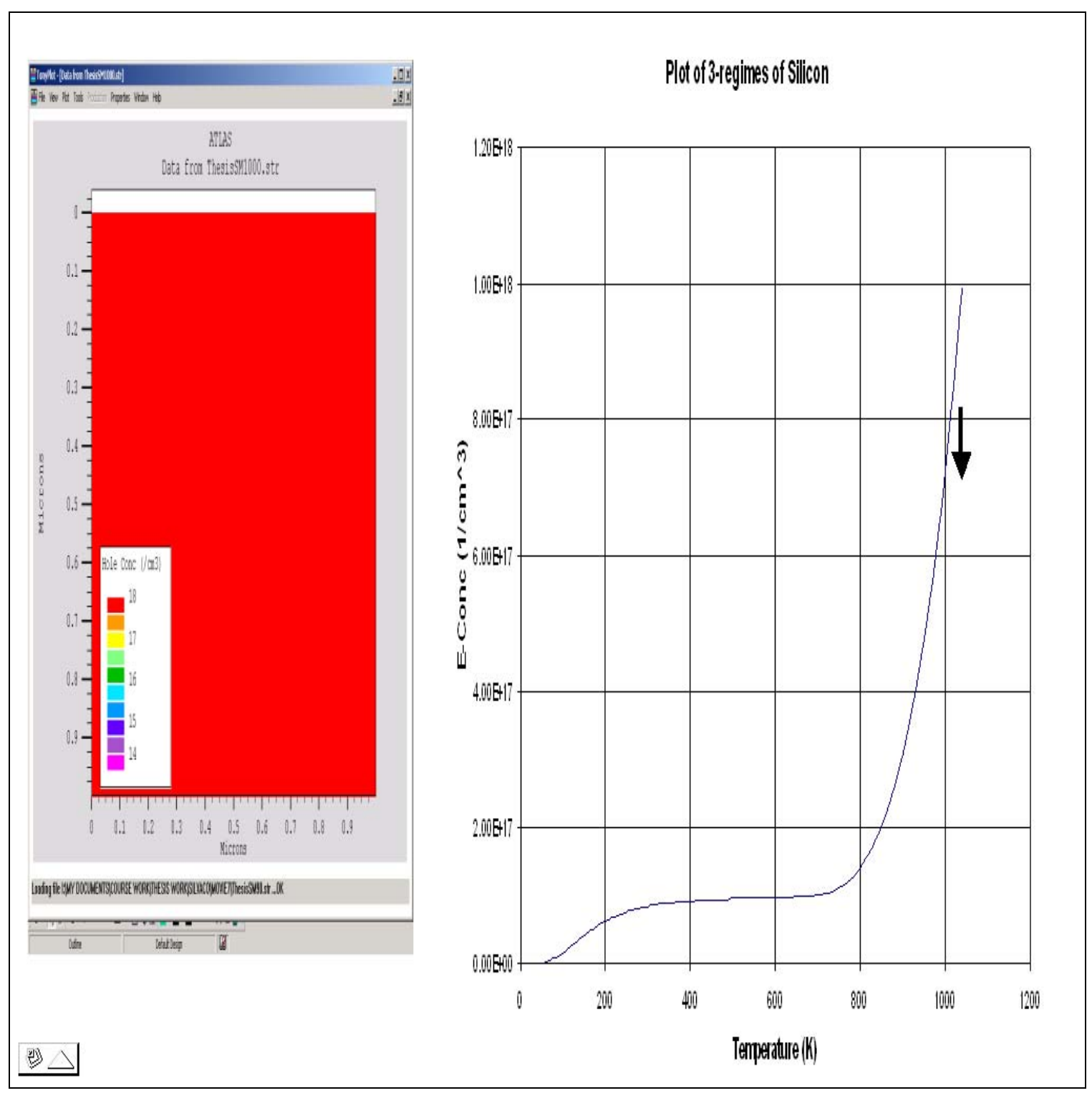

Figure 3-5: Selected Samples of 50 slide Movie $\mathrm{T}=$ $1000^{\circ} \mathrm{K}$ 
THIS PAGE INTENTIONALLY LEFT BLANK 


\section{RESULTS ANALYSIS AND COMPARISON}

\section{A. CHAPTER INTRODUCTION}

This chapter provides a comparison of the results for various approaches obtained from examples taken of Silicon and GaAs. The different conditions and cases documented will be discussed. For this project, silvaco suite was used to develop a procedure to predict temperature dependence of majority carrier concentration. This was discussed in some detail in the previous chapter. The results from Silvaco are compared with MATLAB-based analytic and numerical predictions. The data from both simulations are then compared graphically in Microsoft Excel. The following sections will discuss the processes developed in the simulation to generate the data for this comparison testing .

\section{B. RESULTS FOR SILICON}

Figure 4-1 contains six plots of $\log _{10}\left(p_{0}\right)$ versus $1000 *(1 / T)$ for various conditions from the various sources (numerical, analytic, and Silvaco). The data was also grouped into the case with and without bandgap narrowing (BGN). The model for bandgap narrowing [6] used here is:

$$
E_{g}(T)=E_{g \circ}-\frac{\alpha T^{2}}{(T+\beta)} .
$$

Numbers codes for the various curves on Figure 4-1 appear with the description in the legend. Recommended values $[2,15]$ for silicon, including BGN parameters $\alpha$ and $\beta$, appear in Table 4-1. Note, as expected, curves from the MATLAB- 
based numerical and analytic methods are indistinguishable. For example, MATLAB-based analytic curve \#4(w/BGN) overlays MATLAB-based numerical curve \#6(w/BGN). Furthermore, the impact of BGN is apparent in the curves at the higher temperatures.

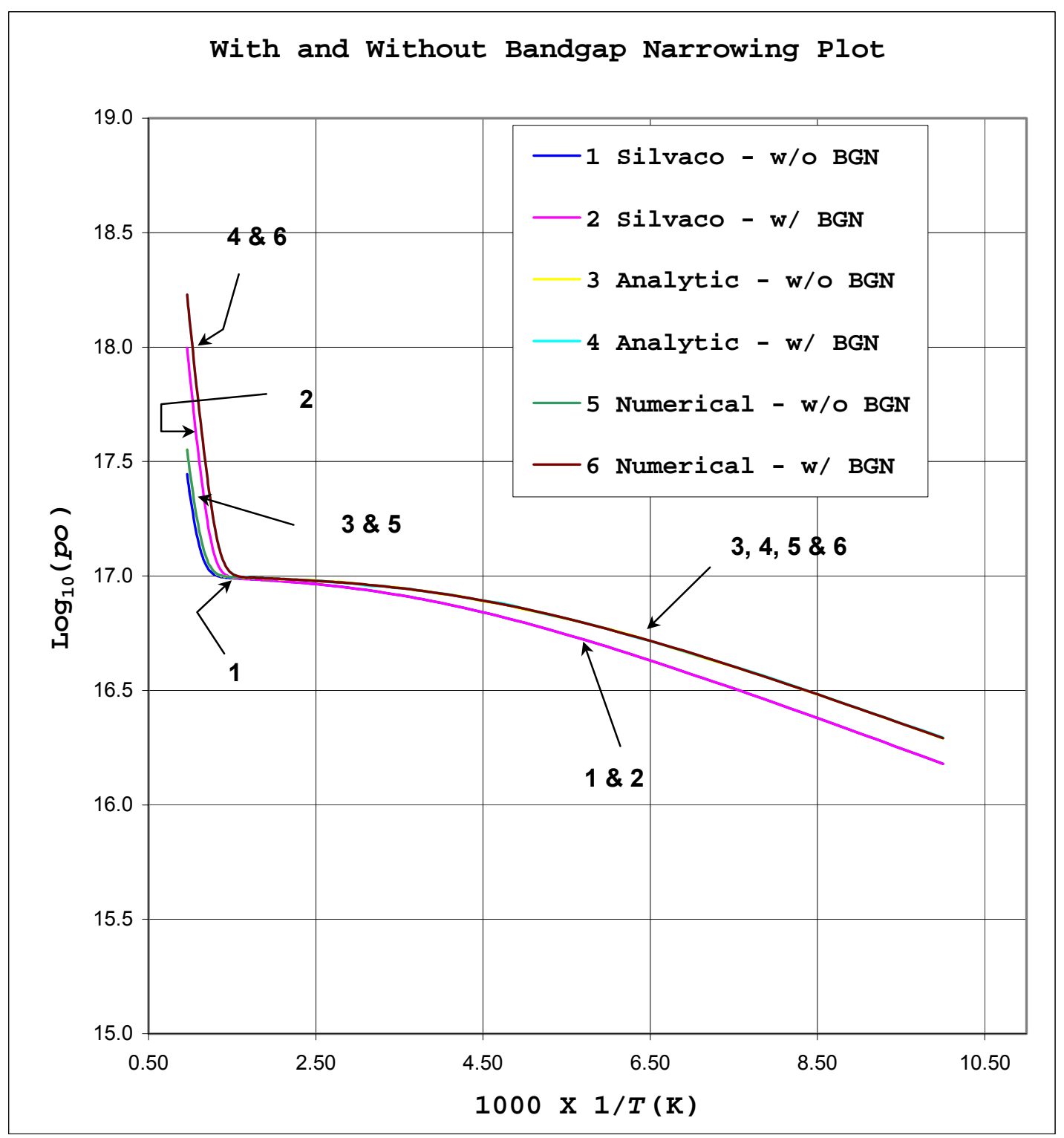

Figure 4-1. $\quad$ Composite Log Plot of three Regimes of Silicon. 
The difference between Silvaco predictions and the one-dimensional modeling efforts are slight but noted for future investigation.

\begin{tabular}{|l|l|}
\hline Parameter & Value \\
\hline$E_{a}$ & $0.045 \mathrm{eV}$ \\
\hline$N_{a}$ & $10^{17} \# / \mathrm{cm}^{3}$ \\
\hline$E_{g o}$ & $1.11 \mathrm{eV} \mathrm{(silicon)}$ \\
\hline$g_{a}$ & 4 \\
\hline$B G N \quad \alpha$ & 0.00047 \\
\hline$B G N \quad \beta$ & 636.0 \\
\hline
\end{tabular}

Table 4-1. Silicon Parameters.

\section{RESULTS FOR GALLIUM ARSENIDE}

The results for GaAs seen in Figure 4-2 were similar to results appearing in Figure 4-1. The parameters used for this test are provided in Table 4-2 [2, 15]:

\begin{tabular}{|l|l|}
\hline Parameter & Value \\
\hline$E_{a}$ & $0.15 \mathrm{eV}$ \\
\hline$N_{a}$ & $10^{12} \mathrm{\# / \textrm {cm } ^ { 3 }}$ \\
\hline$E_{g o}$ & $1.42 \mathrm{eV} \mathrm{(GaAs)}$ \\
\hline$g_{a}$ & 4 \\
\hline$B G N \quad \alpha$ & 0.00054 \\
\hline$B G N \quad \beta$ & 204.0 \\
\hline
\end{tabular}

Table 4-2. GaAs Parameters. 


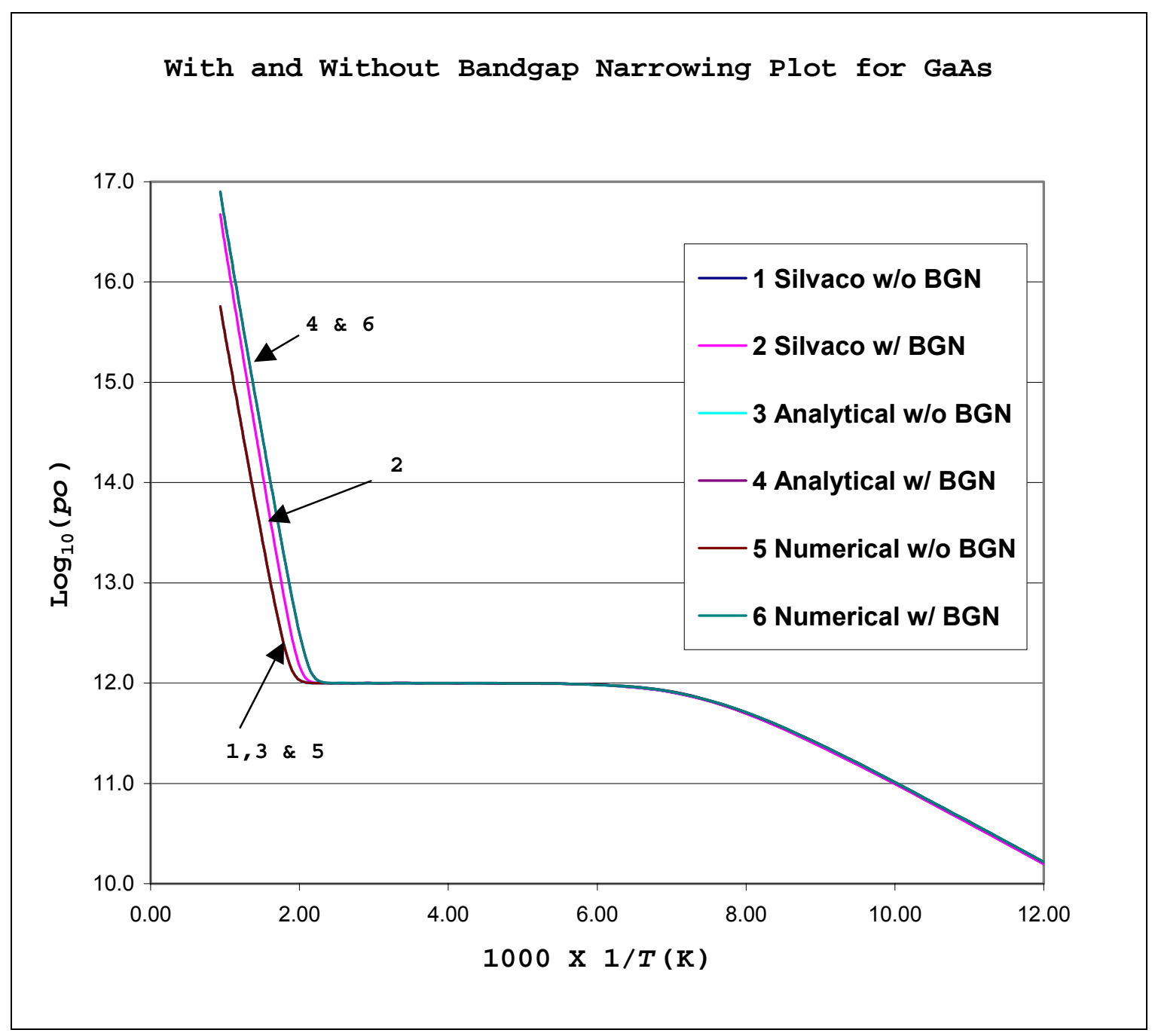

Figure 4-2. Composite Log Plot of three Regimes of GaAs.

Again, all three regimes, i.e., freeze-out, exhaustion, and intrinsic, were evident on Figure 4-2. As before, in the exhaustion regime, the majority carrier was approximately equal to the doping level, i.e., $10^{12} \# / \mathrm{cm}^{3}$. The curves indicated that the MATLAB-based approaches produced predictions that were indistinguishable. It should also be noted that the level of agreement between the Silvaco-based prediction and the MATLAB-based approaches was better in the GaAs case (see Figure 4-2) versus the silicon case (see 32 
Figure 4-1). This is especially true in comparing the level of agreement in the freeze-out regimes of both Figures 4-1 and 4-2. As it turns out, the testing procedure for the two materials, Si and GaAs, was essentially the same with one important exception. For the Silvacobased simulation of Silicon, the input specification for the acceptor energy level $\left(E_{a}\right)$ was not done directly. Instead, the chemical impurity dopant Boron was specified in the DeckBuild code. In the case of the GaAs test, this feature was overridden by a direct specification of the acceptor energy level $\left(E_{a}\right)$ in the DeckBuild code. The latter approach is clearly aligned with the MATLAB-based methods. It is a reasonable conjecture that this accounts for the noticeable improvement in the level of agreement between the Silvaco and MATLAB-based methods.

\section{DISCUSSION OF RESULTS}

The level of agreement between results generated from the MATLAB approaches and from the Silvaco source code tended to confirm the accuracy of the models. The Silvaco and MATLAB-based approaches were in close agreement in predictions for the majority the carrier concentration over all three single impurity regimes of freeze-out, exhaustion, and intrinsic.

An important effect included in the testing was Bandgap Narrowing (BGN) [6]. The results were categorized for each material tested with BGN and without BGN. The differences observed for BGN proved to be fairly significant at higher temperatures. In the exhaustion and freeze-out regimes, the impact of BGN was not noticeable. 


\section{E. CHAPTER SUMMARY}

Both the one-dimensional analytic model and numerical algorithm were essentially indistinguishable in terms of predictions. As expected, when BGN effects were included in the models, the impact was observed at the higher temperatures. The difference between predictions of the Silvaco-based two-dimensional modeling and the onedimensional solutions were observed to be slight but worthy of future investigation. The next Chapter will summarize the main conclusions and will provide suggestions for future work. 


\section{CONCLUSIONS AND RECOMMENDATIONS}

\section{A. SUMMARY OF WORK}

This Chapter provides a summary of the thesis and provides some final thoughts on future areas of study and improvement. The principal completed goal of this research has been to design a convenient automated procedure that will predict, via Silvaco International semiconductor simulation software [1], temperature-dependent exhaustion and freeze-out effects in single-dopant, homojunction semiconductors. To gauge the level of success of this effort the results generated from the Silvaco simulation software have been compared with similar predictions from MATLAB-based analytical and numerical algorithms. This was done for both silicon and gallium arsenide semiconductors.

Chapter I provided an introduction to the objectives and benefits of this research, along with a preview of what topics would be addressed. Chapter II presented an indepth mathematical analysis, which serves as a basis for MATLAB-based analytic and numerical approach. This chapter also detailed the process used to determine the analytical solution. Chapter III was focused on imparting an overview of Silvaco and how it was implemented to provide the necessary results. Chapter IV was used to present the data acquired from the different tests and to make a comparative study based on the data. A sample selection of slides from the Silvaco movie prepared during the thesis is also presented in Chapter IV. 


\section{B. CONCLUSION}

This thesis was designed to be part of a larger study in creating very robust MATLAB-based analytic and numerical simulations to provide an accurate portrait of temperature effects occurring in a semiconductor. This would allow for a versatile and reliable method in predicting the regimes of a semiconductor.

After reviewing the data, it can be concluded that the MATLAB-based analytical and numerical models compared very favorable to the results obtained in Silvaco. All three methods accurately predict the semiconductor behavior in the three regions from freeze-out to the intrinsic. The MATLAB-based analytic and numerical coding allowed for a quick and robust method to generate the majority carrier concentration data of a semiconductor under a varying degree of changes in material and doping level. Currently, the computation cost of results obtained via MATLAB required much less time than the Silvaco method by roughly a factor of 50 .

\section{RECOMMENDATIONS}

There has been significant progress made in this research, but there still exist much additional work to be accomplished in this area. The future of analytical modeling in MATLAB needs to be refined and expanded to allow for a greater range of effects and conditions. For example, the range of cases that can be solved analytically could be widened to include multiple impurities. This capability already exists in the MATLAB-based numerical algorithm. At this present time, the numerical algorithm 
has not satisfactorily been tested for high levels of doping when the Boltzman's approximation fails.

There still exist many conditions and parameters in Silvaco which have not been accounted for which could be implemented in a faster MATLAB model. A more efficient method of handling temperature ranges in Silvaco is also something that needs to be improved upon.

Finally, utilizing Silvaco to create and test a threedimensional model might provide more conclusive data to continue to validate the results obtained for the analytical model. This would provide a significant insight into the limits associated with the one-dimensional models being tested in MATLAB. 
THIS PAGE INTENTIONALLY LEFT BLANK 


\section{APPENDIX A. INTRODUCTION TO GENERAL SEMICONDUCTOR PRINCIPLES (AFTER [11, 12])}

\section{A. QUANTUM MECHANICS}

To fully understand the basis of this thesis, a basic knowledge of quantum effects is required to understand why particles behave as they do at the sub-atomic and atomic dimensional scale. Before the twentieth century, scientists had a firm belief that the physical world was divided into two worlds, the world of particles and the world of waves. This belief was based on the classical system theorized by Sir Isaac Newton.

In the $20^{\text {th }}$ Century, this particle/wave paradigm was challenged and proven to be incomplete, based on the early work of physicist such as Neil Bohr. Werner Heisenberg, and an Austrian, Erwin Schrödinger postulated a new Quantum Theory based on the aforementioned work, and thus a valid and proven method was constructed to provide a means of describing sub-atomic particles.

Quantum mechanics theorizes that the electronic structure of an atom is based on quantum conditions. These conditions are postulated in the theory that electrons in an atom are restricted to certain energy level. This "quantization" defines certain allowable transitions involving absorption and emission of energy by the electrons [11] .

Neils Bohr determined that electrons in orbits moved with well defined momentum. Based on this work and through absorption and emission, an electron may transition to the 
next smaller or larger energy level based on the following rules:

1) Electrons exist in certain stable, circular orbits about the nucleus.

2) The electron may shift to an orbit of higher or lower energy, thereby losing or gaining energy equal to the difference in the energy levels (by absorption or emission of a photon of energy hv, see Figure $A-1)$.

3) The angular momentum $P_{\theta}$ of the electron in an orbit is always an integral multiple of Planck's constant divided by $2 \pi$ [11].

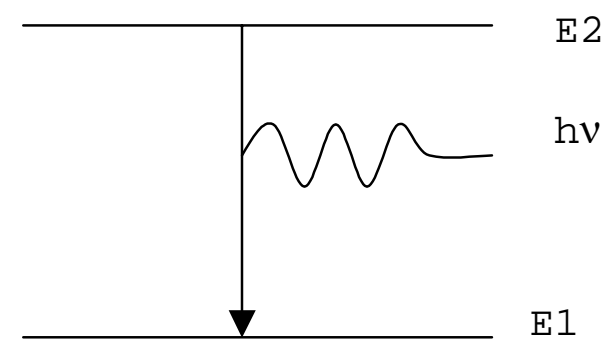

Figure A-1. Emission of Photon.

\section{B. SEMICONDUCTOR MATERIAL PROPERTIES}

A semiconductor is able to conduct electricity at room temperature more readily than an insulator, but less easily than a metal. Electrical conductivity, which is the ability to conduct electrical current under the application of a voltage, has one of the widest ranges of values of any physical property of matter. Such metals as copper, silver, and aluminum are excellent conductors, but such 
insulators as diamond and glass are very poor conductors. At low temperatures, pure semiconductors behave like insulators. Under higher temperatures or light or with the addition of impurities, however, the conductivity of semiconductors can be increased dramatically, reaching levels that may approach those of metals [12].

The common semiconductors include chemical elements and compounds such as silicon, germanium, gallium arsenide, and lead telluride. They increase in conductivity with temperature, light, or impurities arising from an increase in the number of conduction electrons, which are the carriers of the electrical current. In a pure, or intrinsic, semiconductor such as silicon, the valence electrons, or outer electrons, of an atom are paired and shared between atoms to make a covalent bond that holds the crystal together. These valence electrons are not free to carry electrical current. To produce conduction electrons, temperature or light is used to excite the valence electrons out of their bonds, leaving them free to conduct current. Deficiencies, or "holes," are left behind that contribute to the flow of electricity. (These holes are said to be carriers of positive electricity.) This is the physical origin of the increase in the electrical conductivity of semiconductors with temperature. The energy required to excite the electron and hole is called the energy gap.

For a semiconductor, this energy gap is termed the bandgap. It is a forbidden region in which an electron cannot exist, as it is the "space" between the quantized states. The upper region is called the conduction band and 
the lower is labeled the valence band. This separation between the energy of the two bands called the bandgap is labeled as $E_{g}$. It is the most important parameter when dealing with semiconductors since it is the basis for any utilization of a given material. These bandgaps define the relative ease to "free" electron through excitation from thermal, optical, or electrical energy. Semiconductors have lower bandgaps (typically 0.5-4 eV) while insulators have higher bandgaps (5-10 eV) of transition.

Semiconductor devices have many varied applications in electrical engineering. Recent engineering developments have yielded small semiconductor chips containing millions and in the near future, hundreds of millions, of transistors. These chips have made possible great miniaturization of electronic devices. More efficient use of such chips has been developed through what is called complementary metal-oxide semiconductor circuitry, or CMOS, which consists of pairs of $\mathrm{p}$ - and $\mathrm{n}$-channel transistors controlled by a single circuit.

\section{CONDUCTION}

Certain critical terms and concepts must be introduced to better understand the fundamental of semiconductors. Two parameters in the handling and usage of semiconductors are temperature and doping. These two factors are extremely important in determining how effect a semiconductor is and how it can most effectively be used in any system design.

Doping is a method to produce free carriers of electricity in a semiconductor by adding impurities to, or to 
"dope," the semiconductor. The difference in the number of valence electrons between the doping material, or dopant (either donors or acceptors of electrons), and host gives rise to negative (n-type) or positive (p-type) carriers of electricity. This concept is illustrated in the accompanying diagram of a doped silicon (Si) crystal. Each silicon atom has four valence electrons (represented by dots in Figure A-2); two are required to form a covalent bond. In n-type silicon, atoms such as phosphorus (P) with five valence electrons replace some silicon and provide extra negative electrons. In p-type silicon, atoms with three valence electrons such as aluminum (Al) lead to a deficiency of electrons, or to holes, which act as positive electrons. The extra electrons or holes can conduct electricity.

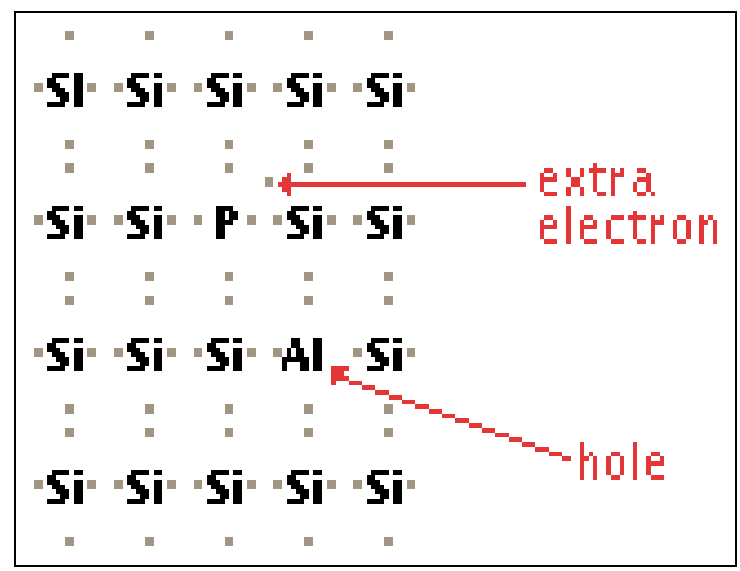

Figure A-2. Si Material Doped with P (From [13]).

When $\mathrm{p}$-type and $\mathrm{n}$-type semiconductor regions are adjacent to each other, they form a semiconductor diode, and the region of contact is called a $\mathrm{p}-\mathrm{n}$ junction. (A diode is a two-terminal device that has a high resistance 
to electric current in one direction but a low resistance in the other direction) The conductance properties of the $\mathrm{p}-\mathrm{n}$ junction depend on the direction of the voltage, which can, in turn, be used to control the electrical nature of the device. Series of such junctions are used to make transistors and other semiconductor devices such as solar cells, $\mathrm{p}-\mathrm{n}$ junction lasers, rectifiers, and many others.

Temperature is also very important and as it is raised from $0^{\circ} \mathrm{K}$, electrons are excited from the thermal energy and they move across the bandgap from the valence band to the conduction band. The resulting material is one with some unoccupied states in the valence band and this vacancy creates a "hole", or an empty state. This creates an electron-hole pair (EHP) and as these "freed" electrons move through the material, this motion induces an electric field and current flows.

There are two major classes of semiconductors, intrinsic and extrinsic. An intrinsic semiconductor is one in which the electrical properties of device is determined by the host material, such as with bulk silicon. In an extrinsic semiconductor, the chemical impurities used to dope the host material are the determining factor for the electrical properties of the material. These dopants can drastically modify the electrical properties of a device as with gallium arsenide. 


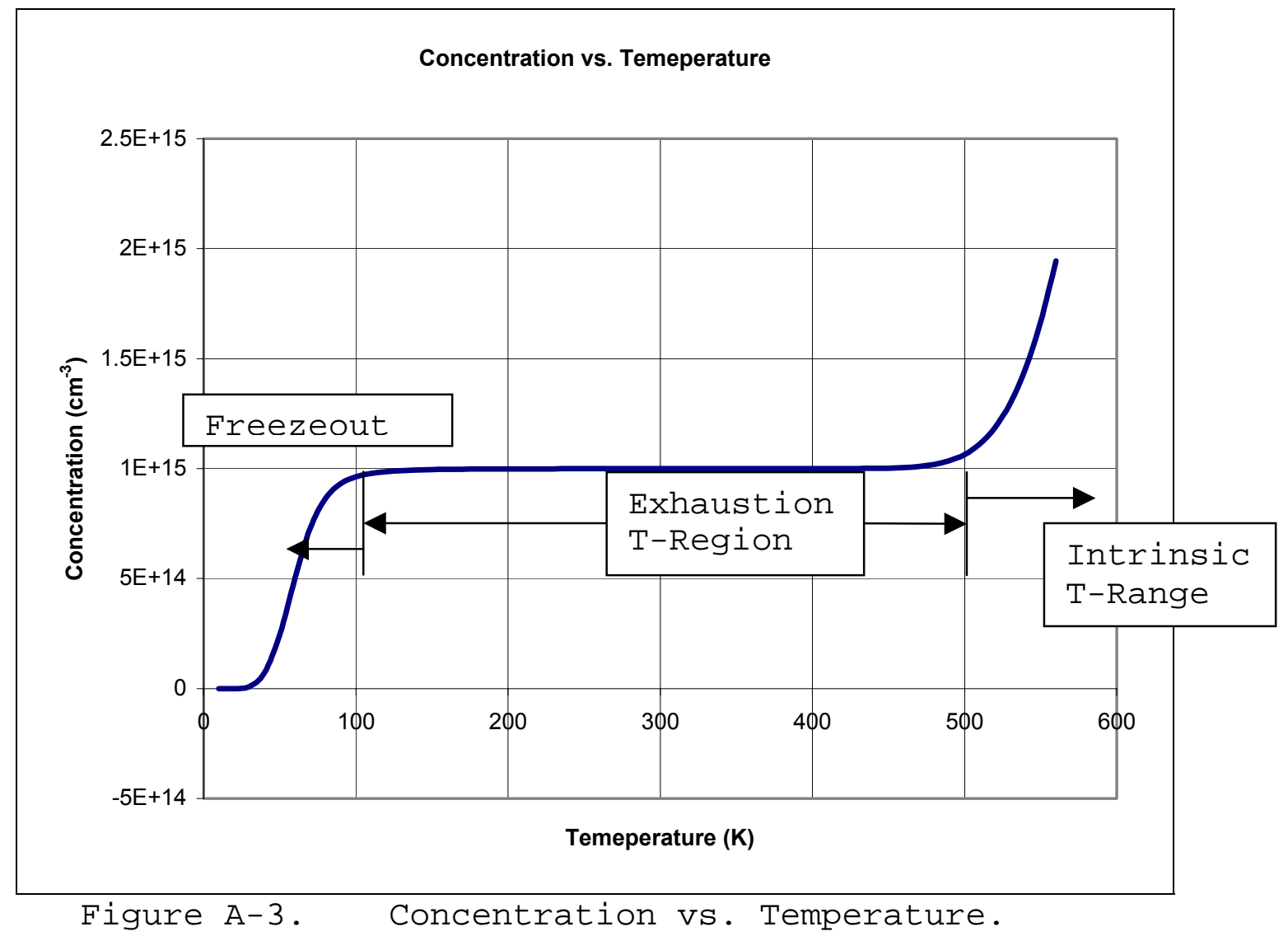

As illustrated in Figure A-3, when the temperature increases, the intrinsic properties of the material override any level of doping applied to the material. This region varies based on material and doping level of impurities. At low temperatures, the energy from thermal effects is not high enough to fully activate all the donor and acceptor impurities. This region is known as the freeze-out region and this topic will be addressed later in this report.

If temperature increases above $0 \mathrm{~K}$, excitation of electrons is induced and EHPs are formed as thermally excited electrons cross the bandgap to the conduction band. The positions of the free electron and hole in the crystal 
are spread out over several lattice spacing and can be considered quantum mechanically by probability distributions. At a given temperature, there is a certain amount of EHPs. In a steady state condition the generation rate $\left(G_{i}\right)$ of EHPs in an intrinsic material must be equal to the rate of recombination of electrons and holes [11].

As temperature increases, the probability distribution of an electron in a range of allowed energy at equilibrium follows Fermi-Dirac statistics, because they obey the Pauli exclusion principal. The Fermi-Dirac distribution function is equal to:

$$
f(E)=\frac{1}{1+e^{\left(E-E_{F}\right) / k T}}
$$

where $E_{F}$ is called the Fermi Level. For intrinsic materials, the probability an electron is at the Fermi level is very near $1 / 2$. When $T=0 K$, this function is a unit step and, as $T$ increases, the function takes on a more curved appearance (see Figure A-4).

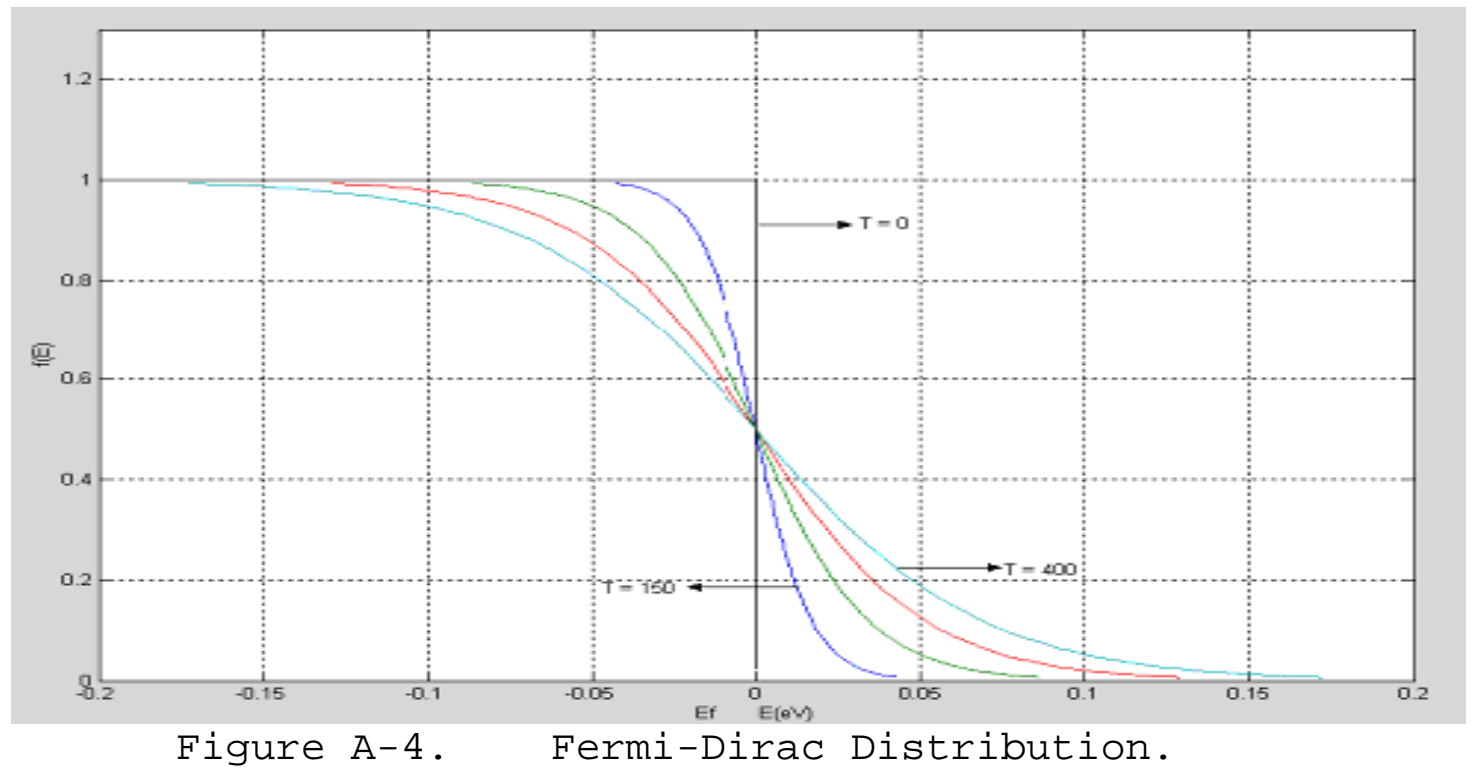




\section{APPENDIX B. OPERATIONAL GUIDE TO REPRODUCING THE RESULTS IN SILVACO}

\section{A. OPERATIONAL GUIDE TO REPRODUCING SILVACO DATA ACQUISITION CAPABILITIES}

Silvaco's semiconductor software is a powerful and very capable tool in deciphering the electrical and material properties of a semiconductor. The vast array of built-in functions and add-on modules create a powerful tool, albeit a tool with a steep learning curve. This concise section will attempt to help create a standardize method to collect, organize, and extract the data from Silvaco for further analysis.

The process begins with the DeckBuild graphical user interface and the creation of an input file. ATLAS is the module used in this research and the module is adequate and effective for all of the work that was accomplished. The physical parameters and structural dimensions were set for the input file; to be used globally throughout the model, in conjunction with a doping level and MODEL command setting. In the MODEL command, the lattice temperature is set for the ATLAS run. At this point, the program is ready to enter the recording stage of the process.

The $P R O B E$ function was used extensively to gather the data required for proper analysis. This function was used to record the temperature, the hole concentration, and the electron concentration. An iterative stepping of minimal bias is applied to allow for the software to calculate the properties of the material. The STRUCTURE (.STR) file is saved and the probed values are logged into a LOG (.log) 
file. At this point, the program is ready to quit the ATLAS run at the given temperature and begin stepping on to the next temperature to begin the process anew. The new temperature is set in the MODEL command. The LOG file is then appended to record the new data from the updated temperature. The process repeats itself until the QUIT command is called to end the process. This raw data can then be exported into Microsoft Excel or MATLAB to be further categorized and analyzed.

\section{B. DECKBUILD ABBREVIATED SOURCE CODE}

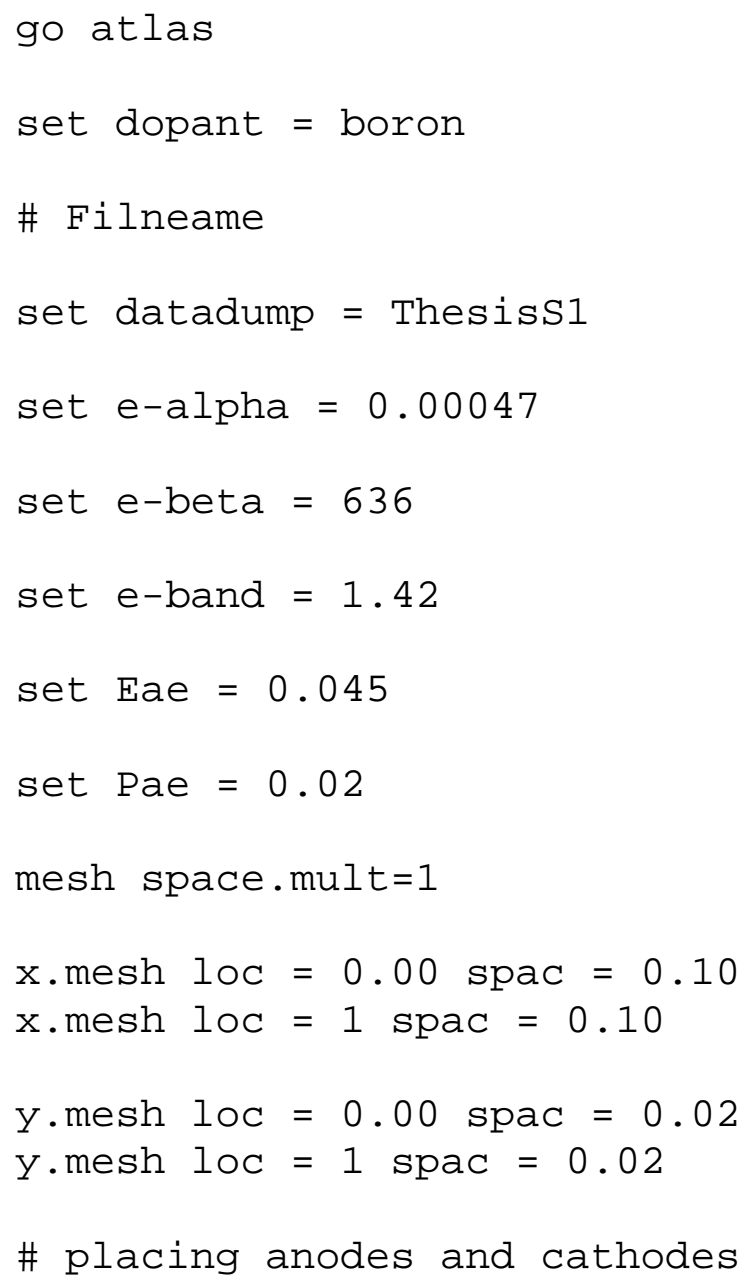




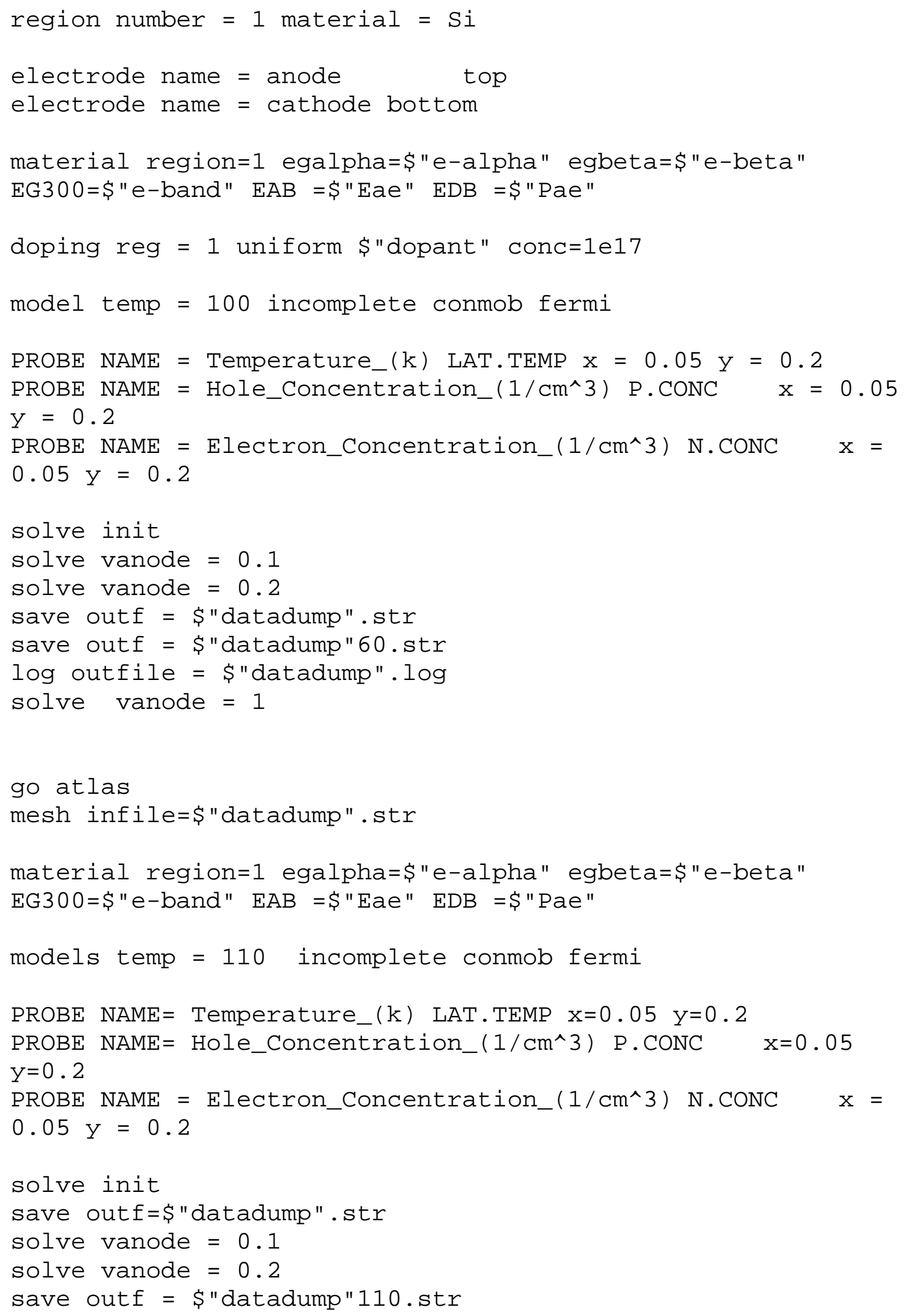




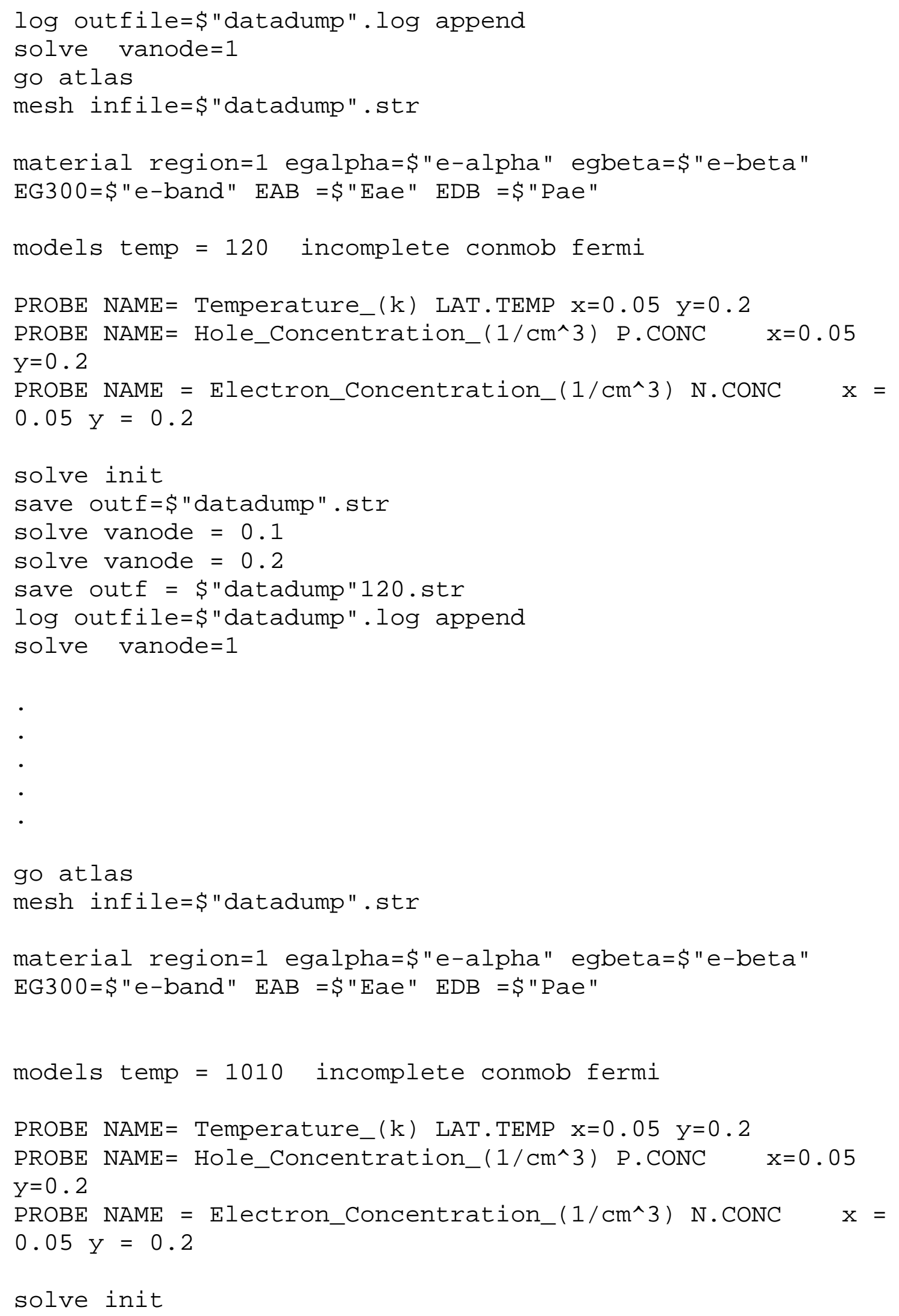




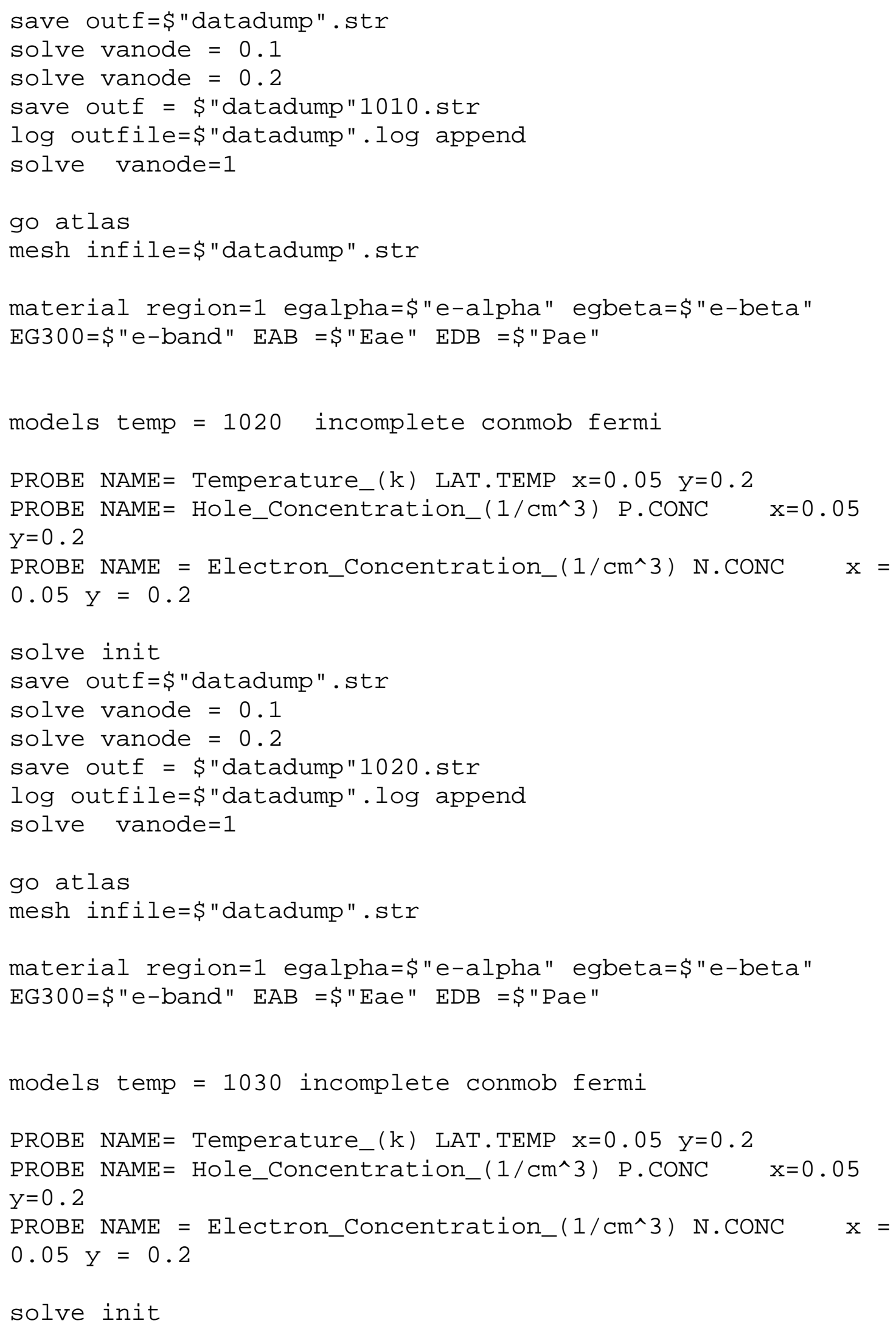




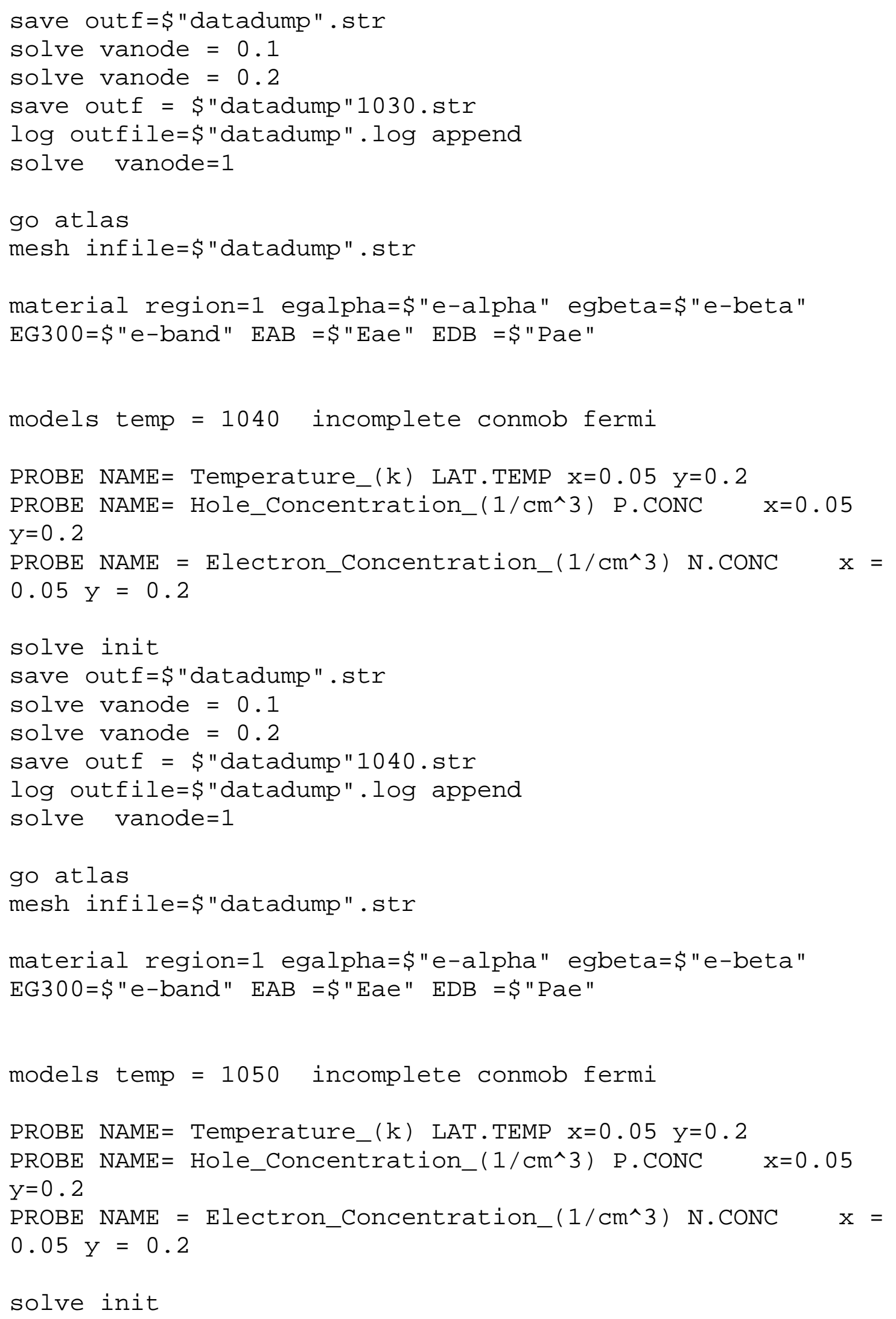


save outf $=\$$ "datadump". str

solve vanode $=0.1$

solve vanode $=0.2$

save outf $=$ \$"datadump"1050.str

log outfile=\$"datadump".log append

solve vanode $=1$

\section{PROCEDURAL GUIDE TO CREATING A MOVIE}

The ability to visualize the effects of temperature on a semiconductor cell is a very impressive method for illustrating the concepts involved. For this purpose, Silvaco has the capability to save STRUCTURE files from the

DeckBuild computations. These files are repositories of information for the semiconductor cell at given conditions. These files can be used to display the carrier concentration, along with a host of other material and electrical properties. To access these files, Silvaco provides a stand-alone viewer called TONYPLOT.

Multiple STRUCTURE files can be loaded into TONYPLOT, and the display drop down menu item can be used to display the carrier concentration of the files. All of these files must be selected because the programs sets the displayed picture relative to the structure windows selected. While there is a effective movie animator built into silvaco, the created movie is not exportable into other media format, thus each individual file must be screen captured into a standard graphical file type such as (.jpg) or (.bmp), for future manipulation. The files can then be organized in commercial moviemaker software and formatted for further animation. (Microsoft Office PowerPoint was used to animate the slides into a movie.) The process is labor intensive, 
but very effective in creating an illustrative tutorial of the desired simulation effect. 


\section{APPENDIX C. PAPER PRESENTED AND ACCEPTED AT THE $45^{\text {TH }}$ IEEE INTERNATIONAL MIDWEST SYMPOSIUM ON CIRCUITS AND SYSTEMS IN TULSA, OK ON AUG 4-7, 2002}

This appendix contains the "Comparison of Analytic and Numerical Models with Commercially Available Simulation Tools for the Prediction of Semiconductor Freeze-out Exhaustion" paper from the 4-7 August $200245^{\text {th }}$ IEEE Midwest Symposium on Circuits and System at Oklahoma state University in Tulsa, Oklahoma. The paper was a condensed report on the work researched in this thesis. 


\title{
Comparison of Analytic and Numerical Models with
}

\author{
Commercially Available Simulation Tools for the Prediction \\ of Semiconductor Freeze-out and Exhaustion
}

\author{
Ron Pieper, Sherif Michael, Derek Reeves \\ Naval Postgraduate School \\ Department of Electrical and Computer Engineering \\ Monterey CA 93943
}

Email: rjpieperanps.navy.mil

\begin{abstract}
Currently, commercial software packages, such as available through silvaco International[1], are well designed to solve the electron/hole transport problem. This type of calculation is usually required to predict the device IV characteristic. Surprisingly, using the same package, to obtain a temperature dependent plot for majority carrier concentration for a uniform semiconductor requires a somewhat complicated procedure [2]. Our paper will present an efficient novel way of obtaining this curve from the Silvaco International software and compare the results with a proposed one dimensional singleequation analytic model and a numerical model that predict the temperature dependence for majority concentration in all regimes [3] [4].
\end{abstract}

\section{Introduction}

Integrated Circuits are specified to operate between designated temperature limits. The circuit designer selects the doping level or levels and typically assumes that the dopants are approximately 100\% ionized, i.e. exhaustion and the temperature are not too high. There can be a significant impact on the value of a plethora of device parameters such as depletions widths and/or FET threshold voltages if the assumption is violated. In the domain that the temperature is too low the percentage ionization of dopant or dopants will be significantly less than 100\%. The value for the majority carrier concentration is depressed significantly below the design value. On the other hand if the temperature is too high the thermal generation effect causes the majority carrier concentration to become excessively higher than the design value in what is called the intrinsic temperature regime. The exhaustion regime lies between this two extremes, intrinsic and freeze-out. It is well known that for a multiple impurity dopant process this "simple" three-regime description can be inadequate. What is important to the designer is the plot of the majority carrier versus the 
temperature, or what is more commonly done, a plot of majority carrier concentration versus reciprocal of the temperature.

To-date most "analytic" methods for determining dominant features in such plots make use of multiple ad-hoc arguments, which taken one at a time, applies in only two of the three regimes mentioned above. [5, 6, 7] Numerical methods based on one-dimensional analysis are applicable and provide significant flexibility in terms of making predictions when there are multiple dopants and when taking into account $2^{\text {nd }}$ order effects. [4] It can be argued that numerical methods are generally going to be applicable over a wider range of problems than what can be solved with exact analysis. Two and three dimensional numerical finite element methods provided in CAD based commercial packages have been widely employed by the engineers in the semiconductor processing industry. The 1-D results both numerical and analytical are compared with predictions from such a package of tools from Silvaco Int. [1] See Figure (1). For details on numerical algorithm please see references [3] and [4].

\section{Basic Analysis}

The physical basis [1] for analysis assumes that there is sufficient spatial uniformity in the doping profiles in order to apply the condition of local charge neutrality,

$$
P(T)=p_{o}-n_{o}-\sum N_{a}^{-}+\sum N_{d}^{+}=0
$$

where $T$ is the Kelvin temperature, $p_{0}$ and $n_{\circ}$ are the equilibrium hole and electron carrier concentrations respectively, while $N_{a}^{-}$and $N_{d}^{+}$ are the ionized acceptors and donors atom concentrations respectively. The summations run over the numbers of impurities. The approach presented is facilitated by defining the parameter $\mathrm{Z}$ where:

$Z=\exp \left[\left(E_{F i}-E_{F}\right) / k T\right]$

where $\mathrm{k}$ is Boltzman's constant, $E_{F}$ is the Fermi level and $E_{F i}$ is the intrinsic Fermi level. In non-degenerate cases, i.e. where the Boltzman approximation can be applied to simplify the exact expression for the carrier concentrations, the $Z$ parameter is directly proportional to the equilibrium hole concentration. The constant of proportionality is the temperature dependent intrinsic concentration. It turns out that Eq. (1) can be expressed as P(Z). Numerical methods based on successive substitution in solving for the zeros in expression (1) were found to be unreliable in producing a convergent solution. However the method proposed here is derived from the numerical scheme known as interval bisection [8] and it was found work well with variety of combinations of profiles and other nonstandard conditions

The effective density of states, which is derived in most introductory semiconductor texts, is given by:

$N_{c}=2 \frac{\left(2 \pi m_{n}^{*} k T\right)^{3 / 2}}{h^{2}}$

$N_{\mathrm{v}}=N_{c}\left(\frac{m_{p}^{*}}{m_{n}^{*}}\right)^{3 / 2}$

where $m^{*}$ is the effective mass for the respective bands [5]. In order to facilitate casting 
Eq. (1) into a form dependent on the $\mathrm{Z}$-parameter the well-known relations for intrinsic concentration and Fermi level are used.

$n_{i}=\sqrt{N_{c} N_{\mathrm{v}}} e^{-\frac{E_{g}}{2 k T}}$

$E_{F i}=\frac{E_{c}+E_{v}}{2}+k T \ln \left(\frac{N_{\mathrm{v}}}{N_{c}}\right)$

Following the standard methods $[5,6,7]$ of expressing carrier concentrations in terms of intrinsic parameters Eqs (4-5), it follows that:

$n_{o}=\frac{n_{i}}{Z}$

$p_{o}=n_{i} Z$

Making use of the Fermi probability distributions [5] to predict ionization levels of the donors and acceptors, i.e., $N_{a}^{-}$and $N_{d}^{+}$, and substitution of Eqs (6) and Eq. (1) and then dividing by the intrinsic concentration [3] leads to a condition on $\mathrm{P}(\mathrm{Z}, \mathrm{T})$ :

$$
P(Z)=0=Z+\sum_{l=1}^{M_{d}} \frac{\bar{N}_{d_{l}}}{1+K_{d_{l}} Z^{1}}-Z^{-1}-\sum_{l=1}^{M_{a}} \frac{\bar{N}_{a_{l}}}{1+K_{a_{l}} Z}
$$

in which the over-bar notation indicates division by $\mathrm{n}_{i}$, and the temperature dependent constants,

$K_{d_{l}} \equiv g_{d} e^{\left(E_{F i}-E_{d_{l}}\right) / k T}$

$K_{a_{l}} \equiv g_{a} e^{\left(E_{a_{l}}-E_{F i}\right) / k T}$

are needed to characterize partial ionization. Also $M_{a}$ and $\mathrm{M}_{\mathrm{d}}$ are the numbers of impurity components, and $g_{a}$ (4 typical for silicon) and $g_{d}$ (2 typical for
Silicon) are the several occupation degeneracy, for acceptors and donors, respectively. The donor and acceptor concentrations with the over-bars are normalized by the intrinsic concentration. The energy levels for the impurities $E_{a}, E_{d}$ are defined relative to the valence band, i.e.,

$E_{d}=E_{c}-E_{d}^{\prime}$

where $E_{d}^{\prime}$ is the standard cited value for donors measured with respect to the conduction band. This analysis will also be the basis for an analytic description provided in the next section.

\section{Analytic Solution, single impurity}

Situation is described by the special case that $M_{a}=1$ and $M_{d}=0$ the summation subscript in Eq (7) can be dropped to lighten the notation. Straightforward algebra leads to a cubic equation in $\mathrm{Z}$.

$Z^{3}+\frac{1}{K_{a}} Z^{2}-\left(\frac{\bar{N}_{a}}{1+K_{a}}\right) Z-\frac{1}{K_{a}}=0$

The corresponding result for a single impurity $\mathrm{N}$-type is exactly the same form, producing a cubic in $Y=1 / Z$ with revised coefficients obtained by letting the ' $a$ ' subscript (for acceptor) be replaced with the ' $d$ ' subscript (for donor). The solution can be defined in terms of coefficients for the reference cubic equation:

$x^{3}+a_{1} x^{2}+a_{2} x+a_{3}=0$

To facilitate representation of the solution the following intermediate parameterization of 
the problem is commonly taken [9].

$$
\begin{aligned}
& Q=\left(3 a_{2}-a_{1}^{2}\right) / 9 \\
& R=\left(9 a_{1} a_{2}-27 a_{3}-2 a_{1}^{3}\right) / 54 \\
& D \equiv Q^{3}+R^{2}
\end{aligned}
$$

where, D, which is referred to as the "discriminant" for the cubic problem will dictate the type of solutions possible. As per the fundamental theorem of algebra there will be three roots. For D negative all roots are real while for $D$ positive only one root is real while the other two are complex conjugate. If $D$ is zero there will be repeated root. For the problem being evaluated it can be shown using symbolic mathematical methods that $Q$ and $D$ will always be negative. Furthermore it can then be shown that of the three roots only one is positive and therefore physically acceptable. That root will be predicted by the following recipe [9]

$$
Z=x=2 \sqrt{-Q} \cos (\theta / 3)-\frac{1}{3} a_{1}
$$

where $\theta=\cos ^{-1}\left(R / \sqrt{-Q^{3}}\right)$. Once $Z$ is determined the majority carrier concentration, $p_{0}$, can be predicted from Eq (6b).

\section{Silvaco Int. based Simulation}

Typically, semiconductor device problems of interest involve conditions such as external voltages and/or spatial nonuniformity in the semiconductor. Under these more general conditions, a solution can be obtained by solving Poisson's equation. This is done with the Silvaco tool known as ATLAS [2]. It is possible to incorporate Fermi-Dirac statistics for purposes of estimating incomplete ionization. Due to lack of space it is not possible to cover all the details in the algorithm involving Silvaco Tools. Here are some critical points. First, the Silvaco predictions are obtained from running the software over 2 dimensional grid of points covering a 2 dimensional, uniform and equilibrium semiconductor. A center point is chosen in the grid and the majority carrier concentration is recorded. This is done in an automated fashion for each temperature in a long list of temperatures covering the range of interest. The Silvaco based recordings described in the next section are obtained via this procedure.

\section{Examples}

Figure 2, which has on it 6 plots of $\log 10 \quad\left(p_{0}\right)$ versus $1000 \times(1 / T)$ for various conditions (with and without band-gap narrowing (BGN) and from various sources (numerical, analytic, and Silvaco). The model for band-gap narrowing [6] used here is.

$E_{g}(T)=E_{g o}-\frac{\alpha T^{2}}{(T+\beta)}$

The various curves are coded by number with the description in the legend. In Table 1 recommended values for Silicon including $B G N$ parameters $\alpha$ and $\beta$ are provided. Note, as expected, curves from the numerical and analytic methods are indistinguishable. Furthermore, as expected the impact of $B G N$ is apparent in the curves at the higher temperatures. The difference between Silvaco predictions and the 1-dimensional modeling 
efforts are slight but noted for future investigation.

\begin{tabular}{|l|l|}
\hline Parameter & Value \\
\hline $\mathrm{Ea}$ & $0.045 \mathrm{ev}$ \\
\hline $\mathrm{Na}$ & $10^{17} \mathrm{\# / \textrm {cm } ^ { 3 }}$ \\
\hline Ego & $\begin{array}{l}1.11 \mathrm{ev} \\
\text { (Silicon) }\end{array}$ \\
\hline ga & 4 \\
\hline $\mathrm{BGN} \alpha \quad$ [6] & 0.00047 \\
\hline $\mathrm{BGN} \beta \quad$ [6] & 636.0 \\
\hline Table 1: Parameters used in
\end{tabular}

Simulations

\section{Conclusions}

Both analytic one-dimensional models and numerical onedimensional algorithms are essentially indistinguishable in terms of predictions. As expected, when BGN effects are included in the models the impact was observed at the higher temperatures. The difference between Silvaco predictions based twodimensional modeling and the one-dimensional solutions were observed to be slight but worthy of future investigation.

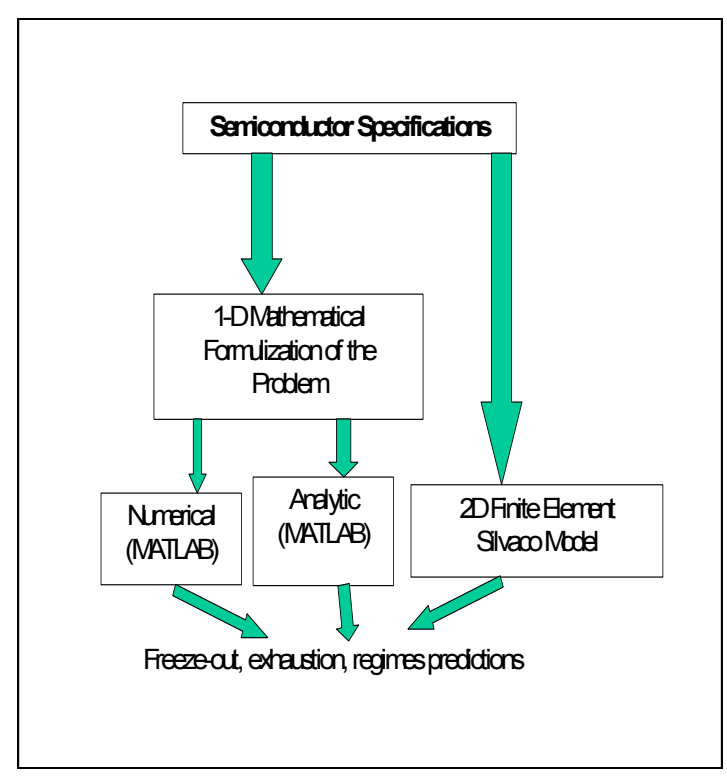

Figure 1: Testing Flowchart

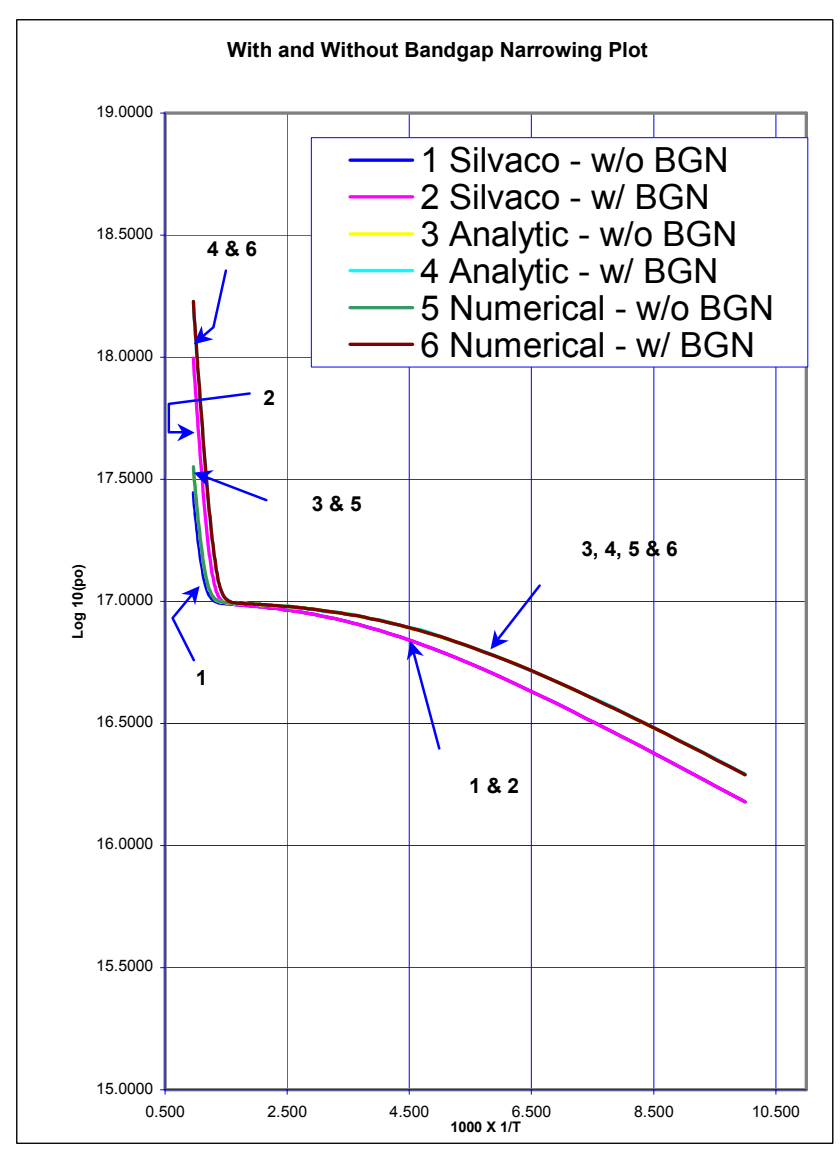

Figure 2: Composite Plot of Results

Acknowledgement :

We appreciate the support provided by the Cryptologic Research Lab at NPS Monterey, $\mathrm{CA}$

References:

[1] Silvaco International, Atlas User's Manual, 4701 Patrick Henry Drive, Bldg1, Santa Clara CA, 94054, Telephone (408) 5671000

[2] "Simulating Impurity Freezeout During Low Temperature Operation" From Silvaco International proprietary journal for process and device engineers, Vol 11, \#11, November 2000

[3] Ron Pieper and Sherif Michael, "A Robust Algorithm for Predicting Freezeout and 


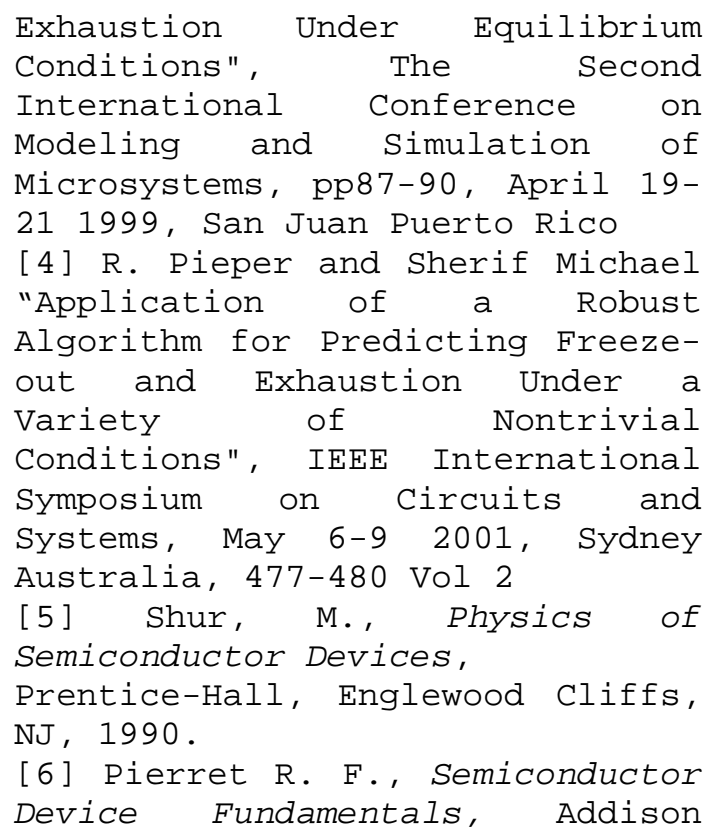

Wesley Publishing Company, New York, 1996, See exercise 2.1 of the text (page 70)

[7] Bar-Lev, A. Semiconductor Electronic Devices, $3^{\text {rd }}$ Edition, Prentice Hall, 1993, (see page 93)

[8] Hamming, R.W., Numerical Methods for Scientists and Engineers, Dover Publications, 1975.

[9] Spiegel, M., Schaum's Outline Series, Mathematical Handbook of Formulas and Tables, McGraw Hill New York 1968, (Fyi There is a typo in this reference's Eq. 9.4 solution for the cubic. Specifically it is missing the term $\left.\left(-1 / 3 a_{1}\right)\right)$. 
THIS PAGE INTENTIONALLY LEFT BLANK 


\section{LIST OF REFERENCES}

[1] Silvaco International, Atlas User's Manual, 4701 Patrick Henry Drive, Bldg1, Santa Clara CA, 94054, Telephone (408) 567-1000.

[2] Andy Strachan (journal editor), "Simulating Impurity Freeze-out During Low Temperature Operation" Silvaco's proprietary journal, Simulation Standard, A Journal for Process and Device Engineers, Vol. 11, \#11, November 2000 .

[3] Ron Pieper and Sherif Michael, "A Robust Algorithm for Predicting Freezeout and Exhaustion Under Equilibrium Conditions", The Second International Conference on Modeling and Simulation of Microsystems, pp. 87-90, San Juan Puerto Rico, April 19-21, 1999.

[4] R. Pieper and Sherif Michael, "Application of a Robust Algorithm for Predicting Freeze-out and Exhaustion Under a Variety of Nontrivial Conditions", IEEE International Symposium on Circuits and Systems, Sydney Australia, Vol. 2, pp. 477-480, May 6-9 2001.

[5] Shur, M., Physics of Semiconductor Devices, PrenticeHall, Englewood Cliffs, NJ, 1990.

[6] Pierret R.F, Semiconductor Device Fundamentals, Addison Wesley Publishing Company, New York, 1996, See exercise 2.1 of the text (page 70.)

[7] Bar-Lev, A., Semiconductor Electronic Devices, 3rd Edition, Prentice Hall, Upper Saddle, NJ, 1993, (see page 93.)

[8] Hamming, R.W., Numerical Methods for Scientists and Engineers, Dover Publications, 1975.

[9] Spiegel, M., Schaum's Outline Series, Mathematical Handbook of Formulas and Tables, McGraw Hill, New York 1968, (There is a typo in this reference's Eq. 9.4 solution for the cubic. Specifically it is missing the term $(-1 / 3 a 1))$. 
[10] Ron Pieper, Sherif Michael, and Derek Reeves, "Comparison of Analytic and Numerical Models with Commercially Available Simulation Tools for Prediction of Semiconductor Freeze-out and Exhaustion, Invited paper presented at the 45th Midwest Symposium on Circuits and Systems, Tulsa OK, Aug 5-7, 2002.

[11] Streetman, B. G., Solid State Electronic Devices, 2nd Edition, Prentice Hall, Englewood Cliffs, NJ, pp. 5290,1980 .

[12] Blakemore, J. S., Semiconductor Statistics, Pergamon Press, Inc., New York, NY, pp. 51-60, 1962.

[13] Encarta Online Encyclopedia, encarta.msn.com/encnet/refpages/artcenter.aspx.

[14] Hanselman, D. and Littlefield, B., Mastering MATLAB 5: A Comprehensive Tutorial and Reference, Prentice Hall, Upper Saddle, NJ, 1998.

[15] Ioffe Physics Technical Institute, Web-based Electronic Archive, "New Semiconductor Characteristics and Properties", www.ioffe.rssi.ru/SVA/NSM. 


\section{INITIAL DISTRIBUTION LIST}

1. Defense Technical Information Center

Fort Belvoir, VA

2. Dudley Knox Library

Naval Postgraduate School

Monterey, CA

3. Chairman, Code EC

Department of Electrical and Computer Engineering

Naval Postgraduate School

Monterey, CA

4. Engineering \& Technology Curricular Office, Code 72 Naval Postgraduate School

Monterey, CA

5. Professor Ron Pieper, Code EC/RP

Department of Electrical and Computer Engineering Naval Postgraduate School

Monterey, CA

6. Professor Sherif Michael, Code EC/MI

Department of Electrical and Computer Engineering

Naval Postgraduate School

Monterey, CA

7. LT Derek E. Reeves

233 Lawson St.

Hempstead, NY 